This item was submitted to Loughborough's Research Repository by the author.

Items in Figshare are protected by copyright, with all rights reserved, unless otherwise indicated.

\title{
Seamless video access for mobile devices by content-aware utility-based adaptation
}

PLEASE CITE THE PUBLISHED VERSION

http://dx.doi.org/10.1007/s11042-012-1120-2

PUBLISHER

(c) Springer

VERSION

AM (Accepted Manuscript)

\section{PUBLISHER STATEMENT}

This work is made available according to the conditions of the Creative Commons Attribution-NonCommercialNoDerivatives 4.0 International (CC BY-NC-ND 4.0) licence. Full details of this licence are available at: https://creativecommons.org/licenses/by-nc-nd/4.0/

\section{LICENCE}

CC BY-NC-ND 4.0

\section{REPOSITORY RECORD}

Nur, Gokce, Hemantha Kodikara Arachchi, Safak Dogan, and Ahmet Kondoz. 2019. "Seamless Video Access for Mobile Devices by Content-aware Utility-based Adaptation". figshare. https://hdl.handle.net/2134/21698. 


\title{
Seamless Video Access for Mobile Devices by Content-Aware Utility-Based Adaptation
}

\author{
G. Nur ${ }^{1}$, H. Kodikara Arachchi ${ }^{2}$, S. Dogan ${ }^{2}$, and A. M. Kondoz ${ }^{2}$ \\ ${ }^{1}$ Kirikkale University, Electrical and Electronics Engineering Departmant, Kirikkale, Turkey \\ gnur@kku.edu.tr \\ ${ }^{2}$ I-Lab Multimedia Communications Research, Centre for Vision, Speech and Signal Processing, \\ University of Surrey, Guildford GU2 7XH, Surrey, UK \\ \{h.kodikaraarachchi, s.dogan, a.kondoz\}@surrey.ac.uk
}

Abstract — Today's Internet multimedia services are characterized by heterogeneous networks, a wide range of terminals, diverse user preferences, and varying natural environment conditions. Heterogeneity of terminals, networks, and user preferences impose nontrivial challenges to the Internet multimedia services for providing seamless multimedia access particularly for mobile devices (e.g., laptops, tablet PCs, PDAs, mobile phones, etc). Thus, it is essential that advanced multimedia technologies are developed to deal with these challenges. One of these technologies is video adaptation, which has gained significant importance with its main objective of enabling seamless access to video contents available over the Internet. Adaptation decision taking, which can be considered as the "brain" of video adaptation, assists video adaptation to achieve this objective. Scalable Video Coding (SVC) offers flexibility for video adaptation through providing a comprehensive set of scalability parameters (i.e., temporal, spatial, and quality) for producing scalable video streams. Deciding the best combination of scalability parameters to adapt a scalable video stream while satisfying a set of constraints (e.g., device specifics, network bandwidth, etc) poses challenges for the existing adaptation services to enable seamless video access. To ease such challenges, an adaptation decision taking technique employing a utility-based approach to decide on the most adequate scalability parameters for adaptation operations is developed. A Utility Function (UF), which models the relationships among the scalability parameters and weights specifying the relative importance of these parameters considering video content characteristics (i.e., motion activity and structural feature), is proposed to assist the developed technique. In order to perform the developed adaptation decision taking technique, a video adaptation framework is also proposed in this paper. The adaptation experiments performed using the proposed framework prove the effectiveness of the framework to provide an important step towards enabling seamless video access for mobile devices to enhance viewing experience of users.

Index Terms - Adaptation decision taking, content-awareness, Utility Function (UF), Mean Opinion Score (MOS), motion activity, Scalable Video Coding (SVC), structural feature, video adaptation. 


\section{INTRODUCTION}

The phenomenal rise of multimedia content on the Internet available to an increasing number of users, who intend to consume it anytime, with different preferences, using particularly a plethora of mobile devices (e.g., tablet PCs, PDAs, laptops, mobile phones, etc) and through heterogeneous networks impose nontrivial challenges to universal access of multimedia content [Carvalho et. al., 2004]. Universal Multimedia Experience (UME) is a strategic concept that has a vision of addressing high user satisfaction despite the existence of diverse mobile device capabilities, different user preferences, varying network and natural environment conditions. Thus, UME can be considered as the future of universal access to the multimedia content provided by the Internet multimedia services [Carvalho et. al., 2004]. Video adaptation, which can enable personalized video access, is an essential process for the Internet multimedia services to achieve the UME vision.

Scalable Video Coding (SVC) provides flexible video representations with different frame sizes, frame rates, and bit rates [Thang et. al., 2004]. Video representations provided by SVC can be adapted to the networks, terminals, and users through video adaptation [Thang et. al., 2004, Eberhard et. al., 2007, Chang et. al., 2005]. Adaptation decision taking, which is a process of deciding the most adequate adaptation parameters to use in adaptation operations while accommodating a set of contextual constraints, can be considered as the "brain" of video adaptation. Therefore, advanced adaptation decision taking techniques have to be developed to assist video adaptation to achieve the UME vision for provision of seamless video access particularly for mobile devices.

There are different approaches in literature to develop advanced adaptation decision taking techniques [Carvalho et. al., 2004, Chang et. al., 2005]. Utility-based adaptation decision taking approach is one of the existing optimizationbased adaptation decision taking approaches, which relies on finding maxima or minima of models designed for decision taking to solve an optimization problem. Optimization problem is the problem of finding the best solution among all feasible solutions. The utility of a video content after undergoing adaptation operations can be expressed with a Utility Function (UF) in the utility-based adaptation decision taking approach. It has to be noted that there is an important difference between the terms utility and quality. Utility refers to the satisfaction of the end-user whereas

quality refers to the quality of the content undergoing adaptation operations [Chang et. al., 2005]. Utility-based approach has been widely used in adaptation decision taking strategies in literature. In [Mukherjee et. al., 2005], a simple UF, which only considers bit rate of video contents and is irrespective of video content characteristics, is used in ad- 
aptation decision taking operations. A utility-based adaptation decision taking technique selecting adaptation parameters considering usage environment constraints for dynamic adaptation of video sequences are proposed in [Sofokleous et. al., 2003]. Frame rate, spatial resolution, and quality scalability parameters are not considered in this technique. In [Wang et. al., 2007] adaptation, resource, and quality aspects of adaptation strategies are modeled with a UF. The UF presented in this study considers the frame rate and spatial resolution parameters, but not the quality parameter in the adaptation operations. In [Chang et. al., 2007] only a conceptual framework modeling the relationships among adaptation (e.g., video skimming, frame dropping, etc), resource (e.g., network bandwidth, display resolution, etc), and quality (e.g., objective or subjective quality of a video content) is developed. The objective of this framework is to find the optimal adaptation operations satisfying the resource constraints for achieving maximum quality for a given video content. In [Prangl et. al., 2007], a UF, which relies on the interrelationship of the spatial resolution, frame rate, and Quantization Parameter (QP), is designed. The importance of the spatial resolution, frame rate, and QP on the UF calculation with respect to each other is computed using weights. A recommendation system is used to maintain feedback from each user to determine the weights of the UF for each user. In [Onur et. al., 2007], a UF that relies on jointly modeling the frame rate and spatial resolution is proposed. In addition, the frame rate and spatial resolution are considered as the functions of hardware capabilities (e.g., Whetstone metric, which is used to indicate the performance of the Central Processing Unit (CPU) of a computing device) and video content characteristics (e.g., low spatial details in the video sequence). Although the frame rate and spatial resolution are taken into consideration, the quality aspect of the video has not been considered in the UF. In [Onur et. al., 2005], a UF, which models the bit rate, spatial resolution, quality of the video contents, the terminal capabilities (i.e., the screen size and the clock speed of the CPU of the terminal), and the transmission channel capacity, is proposed. However, the UF model in [Onur et. al., 2005] does not consider the video content characteristics for deriving adaptation decisions. In [Bocheck et. al., 1999], a UF, which is represented as a function of bandwidth, is proposed. The spatial resolution and frame rate of video contents are not considered in this UF either.

In light of the discussions above, it can be summarized that the existing adaptation techniques relying on a utilitybased approach do not consider either all of the scalability parameters or content-related contextual information in their respective adaptation decision taking operations. Therefore, as the first contribution of this paper, a UF, which models the relationships among the frame rate, spatial resolution, and quality parameters of the scalable video con- 
tents, and the weights specifying the relative importance of these parameters, is proposed to measure the utility of video contents. In this way, the relations of trade-off among all of the scalability parameters are considered in the UF as opposed to the existing video adaptation techniques. Video Quality Metric (VQM) [Wolf et. al., 2002] is used to measure the quality parameter values of the UF due to its correlation with HVS in this work. Determining the values of weights for different video content types is essential to consider content related contextual information in adaptation decision taking operations to enable seamless video access for mobile devices. Thus, as the second contribution of this paper, a Motion Level (ML) and Structural Feature Level (SFL) driven classification algorithms are proposed to categorize video contents into different classes/types depending on their motion activities and structural features. In order to determine the weights for the video sequences belonging to each class, users' reactions towards the variations in the adaptation parameters of video contents are monitored in subjective experiments. The weights are then ascertained by the knowledge gained through the results of the experiments. A weighing factors table is developed using the weights associated with each type, as the third contribution of this paper. In order to perform the proposed adaptation decision taking technique, a video adaptation framework is also proposed in this paper, as the forth contribution. Adaptation Quality of Service (AQoS) and Universal Constraints Descriptor (UCD), which are MPEG-21 Digital Item Adaptation (DIA) supported description tools [Information Technology, 2007, Vetro et. al., 2004], are used to assist the adaptation decision taking process in the framework.

The rest of this paper is organized as follows. The proposed algorithms used for classifying video sequences are presented in Section 2. Section 3 discusses the UF adopted to use in the adaptation decision taking strategy. The subjective tests are presented in Section 4. Section 5 analyzes the results obtained through these subjective tests. The proposed video adaptation framework is introduced in Section 6. Adaptation decision taking algorithm and its results derived to evaluate the performance of the proposed video adaptation framework are discussed in Section 7. Finally, Section 8 concludes the paper.

\section{CLASSIFICATION OF VIDEO SEQUENCES}

Eliminating high spatial, temporal, and quality enhancement layers during adaptation yields different types of visual distortions for different video sequence types (e.g., high motion, low structural feature, etc.) [Akyol et. al., 2007]. Therefore, eliminated high spatial, temporal, and quality enhancement layers during adaptation operations should be 
unique for every sequence type to achieve better video perception of users. For this reason, video sequences should be classified into different types. Finding the prominent features of video sequences is a requirement for accurately classifying them into different types. The features related to temporal, spatial, and quality scalability parameters are taken into account to find the features that can be used for the classification because they are the core adaptation parameters. Temporal scalability allows for different frame rates for the video sequences. The motion activity feature of a video sequence is very important to determine the most adequate frame rate for a video sequence to achieve improved video perception [Akyol et. al., 2007]. Therefore, this feature is used as the prominent feature associated with the temporal scalability to classify the video sequences into different categories. Blurring and blocking artifacts are introduced to the sequences when the sequences are scaled in spatial and quality dimensions [Canny et. al., 1986]. A set of experiments was conducted to find out the most prominent feature that these artifacts affect in a video sequence when it is scaled in spatial and quality dimensions. The details of these experiments will be discussed later. According to the results of the experiments, structural feature is determined as the prominent feature associated with the spatial and quality scalability options. Considering these features, ML and SFL classification algorithms are proposed in this paper.

Other reasons of using the motion and structural feature for classification are that they are generic features and have less number of limitations compared to the other features such as location, brightness, etc. For example, the location feature, which specifies the parts of the frames of a video clip that get more attention from the viewers, could be taken into account while classification. Nevertheless, it has some limitations: it considers that regions near the centre of the frames are typically assumed to get more attention from the viewers [Hsiao et. al., 2007], and thus they can be referred to as the "focus of attention" in a video scene. However, this focus of attention is not applicable to all of the video sequences. For instance, the visual attention is typically on the ball in a football match even if the ball is not in the centre of the scene. Moreover, brightness is another feature that could be taken into consideration for classification, but this feature has also some drawbacks. The viewers' attention is usually attracted by the brighter parts of a scene. However, there are also many exceptions to this assumption. For instance, in video clips that contain a bright sky, even if the sky is the brightest part of the scene, it does not usually draw much of the attention, as it mostly comprises the background of a scene [Hsiao et. al., 2007]. Motion and structural features do not have the drawbacks of the aforementioned visual features that hinder them from becoming generic factors for classification. 


\section{1.}

Motion Level Driven Classification

The pyramidal implementation of Lucas and Kanade [Bouguet et. al., 1999] is used to measure the optical flow [Fleet et. al., 2007] in the video sequences in this paper. Lucas and Kanade [Lucas et. al., 1986] method is one of the most popular versions of optical flow estimations since this method is less sensitive to noise, which appears as visual artifacts (e.g., blurring, blocking etc.) in the images. Additionally, heavy computing cost of global motion estimation process is reduced with this method [Bouguet et. al., 1999, Fleet et. al., 2007, Lucas et. al., 1986]. The pyramidal implementation of the Lucas and Kanade is an iterative version of the Lucas and Kanade optical flow algorithm in pyramidal representations of frames in a video sequence [Bouguet et. al., 1999]. Pyramidal representations are different spatial resolutions of each frame in the sequence. For instance, if the spatial resolution (i.e., width $\mathrm{x}$ height) of the frame is $O \times P$, the pyramidal representations of this resolution are: $O / 2 \times P / 2, O / 4 \times P / 4, O / 8 \times P / 8$, and $O / 16 \times$ $P / 16$. Generally, maximum four pyramidal representations are used in the pyramidal implementation of the Lucas and Kanade algorithm since going beyond four representations is not applicable for most of the typical frame resolutions. The reason behind using pyramidal resolutions is that more consistent motion vectors can be provided by refining the motion vectors at different pyramidal resolutions. Moreover, large motion vectors can be handled using the pyramidal implementation of the Lucas and Kanade algorithm [Bouguet et. al., 1999]. The optical flow is first computed at the very deep pyramidal resolution (e.g., $O / 16 \times P / 16)$ since the search range is smaller in this resolution, and accordingly it does not require large computations. Then, the optical flow computations are propagated to the optical flow calculation of a higher resolution. The motion vectors from the lower resolution are scaled for the higher resolution. This process is repeated up to the original frame resolution (e.g., $O$ x $P$ ) [Bouguet et. al., 1999]. Usually, motion information is not evenly distributed to all parts of an image. Therefore, the prominent points, which can be taken into account in the optical flow measurements, should be determined. Shi and Tomasi algorithm [Tomasi et. al., 1991], which selects the corners of the objects as the prominent points, is used in the optical flow measurements in the paper. After the prominent points are selected, they are tracked from frame to frame in a video sequence by the pyramidal implementation of the Lucas and Kanade algorithm. Let $M V(x, y)$ be the motion vector of a feature point having $x$ and $y$ direction components, the motion intensity of a frame of a sequence is calculated as follows:

$$
\Pi(i)=\sum_{d=1}^{N o P}\left|M V_{d}\left(x_{i}, y_{i}\right)\right|
$$

where $\Pi(i)$ is the motion intensity of the $i^{\text {th }}$ frame of a sequence. $d$ and NoP are a feature point and the number of fea- 
ture points in the frame, respectively. $M V_{d}\left(x_{i}, y_{i}\right)$ is the motion vector of the $i^{\text {th }}$ frame at feature point $d$.

It should also be noted that the motion intensity of a frame is directly proportional to spatial resolution of the frame and inversely proportional to the temporal resolution of the video sequence. The reason is the higher the spatial resolution of the frame the higher the motion intensity of the frame. Moreover, the higher the frame rate of the video sequences the lower the motion intensity of the frame [Shechtman et. al., 2005]. Thus, the normalized average motion intensity over a given video sequence, which is used for ML classification, can be calculated as follows:

$$
M=\frac{\sum_{i=1}^{\mathrm{NOF}} \Pi(i)}{\mathrm{NoF}} \cdot \frac{F}{S}
$$

where, $M$ is the motion value in a sequence. NoF is the number of frames in the sequence, $S$ and $F$ are the spatial resolution of a frame and frame rate of the sequence, respectively.

In order to represent the $M$ results for different video sequences, (2) was tested with 18 video sequences, which have Common Intermediate Format (CIF: 352x288 pixels) resolution and 30 fps. Fig. 1 presents the $M$ results for these sequences. The behavior of $M$ against various spatial and temporal resolutions of a selected sequence (i.e., Soccer) is demonstrated in Fig. 2 to prove that (2) provides similar results for video sequences that have different spatial resolutions (i.e., Standard Definition (SD: 704x576 pixels), CIF, and Quarter CIF (QCIF: 176x144 pixels)) and frame rates (i.e., $7.5,15$, and $30 \mathrm{fps}$ ).

As also reported in [Hsiao et. al., 2007], four different classes are determined to categorize the video sequences considering object and camera activities. The four classes are: low motion, low-medium motion, medium-high motion, and high motion, as illustrated in Table I. In this table, the ML categories are represented with A, B, C, and D with respect to the aforementioned classes. $K$-median clustering method [Mangasarian et. al., 2004] is used to determine the borders for each of the classes. Table I also presents the borders of $M$ values for each of the classes. A video sequence can be classified into one of the classes depending on which border its $M$ value belongs to. 


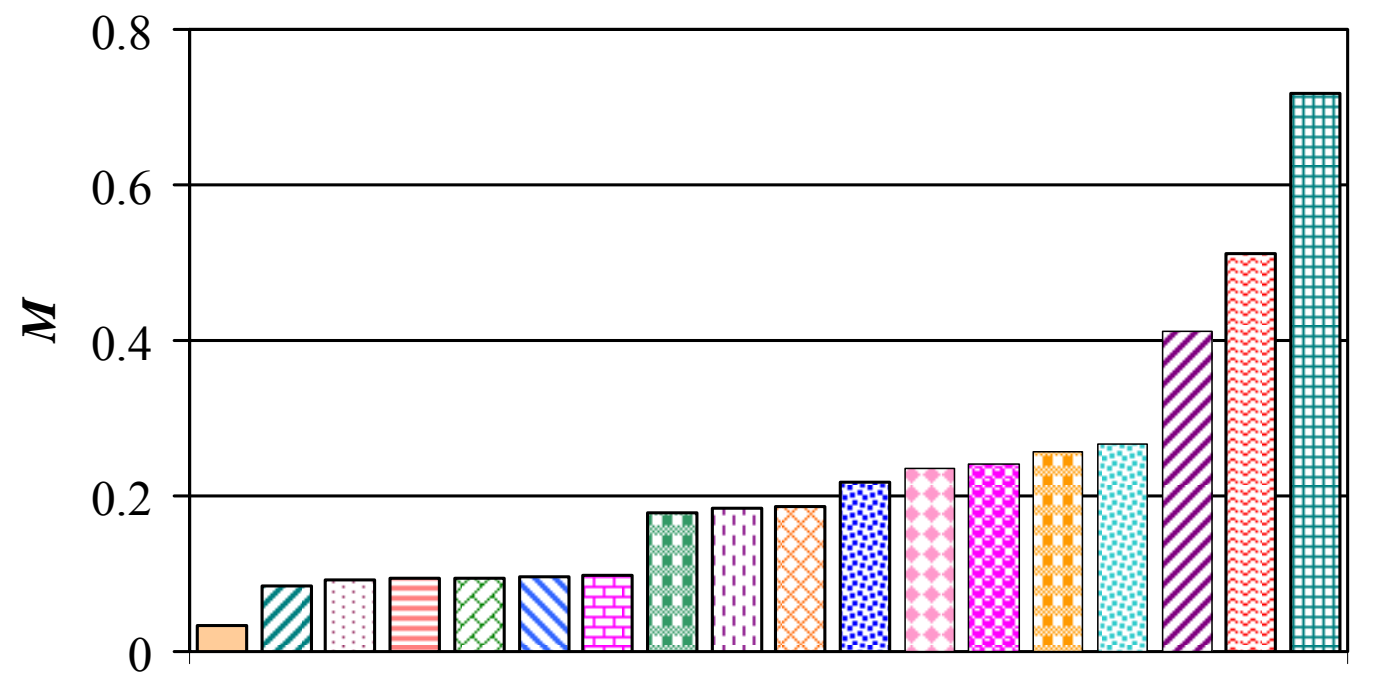

\begin{tabular}{|c|c|}
\hline $\begin{array}{l}\text { ERIC } \\
\text { 昌ILENT } \\
\text { 罗INGER } \\
\square \text { FOREMAN } \\
\text { 羃ITY } \\
\square \text { STEFAN }\end{array}$ & 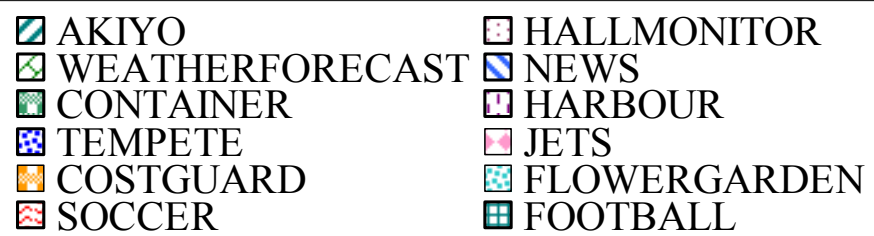 \\
\hline
\end{tabular}

Fig. 1. The measured $M$ results for different video sequences

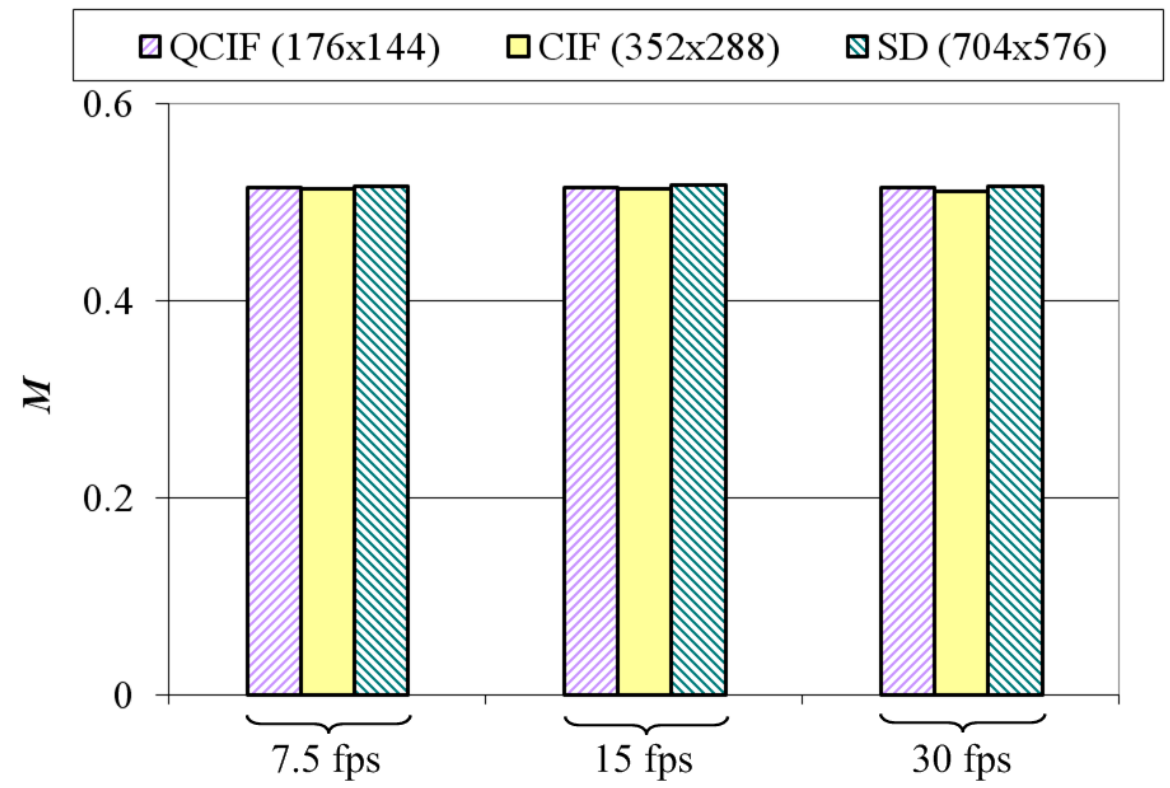

Fig. 2. The $M$ results for the Soccer sequence that has three different spatial resolutions (QCIF, CIF, and SD) and frame rates $(7.5,15$, and $30 \mathrm{fps})$. 
TABLE I

THE MOTION LEVELS

\begin{tabular}{|c|c|c|c|}
\hline $\boldsymbol{M L}$ & Motion Intensity & Camera-Object(s) Motion & $\boldsymbol{M}$ Values \\
\hline A & Low & Static-Static & $0<M \leq 0.1$ \\
\hline B & Low-Medium & Static-Moving & $0.1<M \leq 0.2$ \\
\hline C & Medium-High & Moving-Static & $0.2<M \leq 0.3$ \\
\hline D & High & Moving-Moving & $M>0.3$ \\
\hline
\end{tabular}

\section{2.}

Structural Feature Level Driven Classification

Changing spatial and quality scalabilities result in blurring and blocking effects in the frames, respectively [Akyol et. al., 2007]. The blurriness and blocking effects are generated by spatially down/up sampling the Flower Garden sequence and encoding it with a high QP, respectively, and the results are presented in Fig. 3. As can be observed from the figure, both the blurring and blocking effects influence the objects and background structures in the frames. Thus, the mostly distorted features of structures in the frames when a frame becomes blurry or blocky are determined. Then, these features are used as the basis to classify video sequences associated with spatial and quality scalabilities in the paper. For determining these features, two different experiments were conducted using Structural SIMilarity (SSIM) objective quality measurement technique. SSIM is used in the experiments, because it evaluates the amount of structural distortion of a distorted video compared to the original one [Wang et. al., 2004].

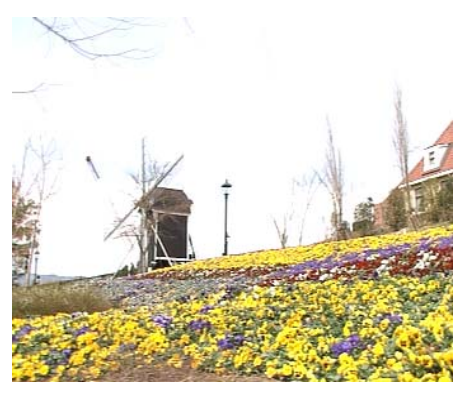

(a)

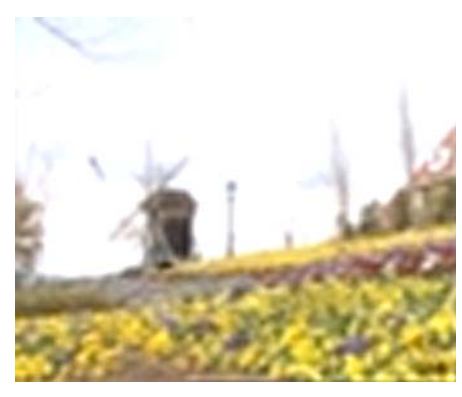

(b)

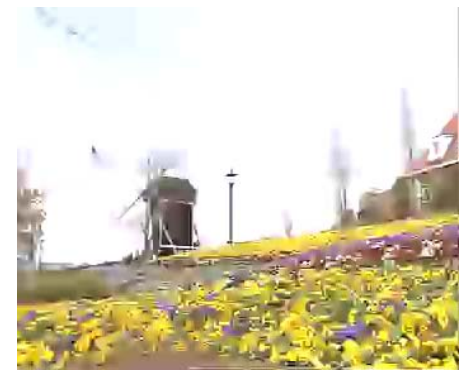

(c)

Fig. 3. Sample (a) original (b) blurry (c) blocky frames of the Flower garden sequence

For the first experiment, different video sequences at CIF resolutions were spatially down sampled to half-QCIF resolution (i.e., $88 \times 72$ pixels) and then up-sampled back to their original CIF resolutions. Next, SSIM of the up-sampled sequences were measured. For the second experiment, different video sequences were encoded with a high QP (i.e., 45), and SSIM of the encoded sequences were measured. Figs. 4 and 5 illustrate the SSIM results of the two experiments using different video sequences. It is observed from the results of each of the sequences distorted with spatial 
or quality scaling that the more the amount of contours in the frames of a video sequence (e.g., Flower Garden, Harbor, etc), the lower the SSIM result of the sequence is. On the contrary, when the frames of a video sequence scaled in spatial or quality dimension contain fewer amounts of contours (e.g., Akiyo, News, etc) the resultant SSIM for these sequences presents high values. Thus, it is envisaged that contour, which characterizes the boundaries of the objects in a frame, is the most notable feature that is distorted when the frame is scaled in spatial and quality dimensions. Therefore, the contour feature, which is associated with the structural feature, has been used as the prominent factor for the classification related to spatial and quality scalabilities. Accordingly, an SFL driven classification algorithm is proposed. For this purpose, the Canny edge detection algorithm [Canny et. al., 1986] was used to determine the contour feature in the frames of a video sequence. The variance in the luminance pixel intensities in the frames is determined by this detection algorithm. Edge detection is performed in five steps by the algorithm. As the first step, the noise in the frames is removed by applying a filter (e.g., Gaussian). Second, the gradient of the frame is determined. Then, the $x$ and $y$ directions of the edges are determined using the gradient. In the fourth step, the determined edge directions are associated to specific directions that can be traced in the frames. As the fifth step, the pixels that are not considered as edges are suppressed, which means that they are set to 0 by tracing them in the edge direction. On the contrary, the pixels that are considered as edges are set to 1 by the Canny algorithm [Grigorescu et. al., 2004, Papari et. al., 2006]. 


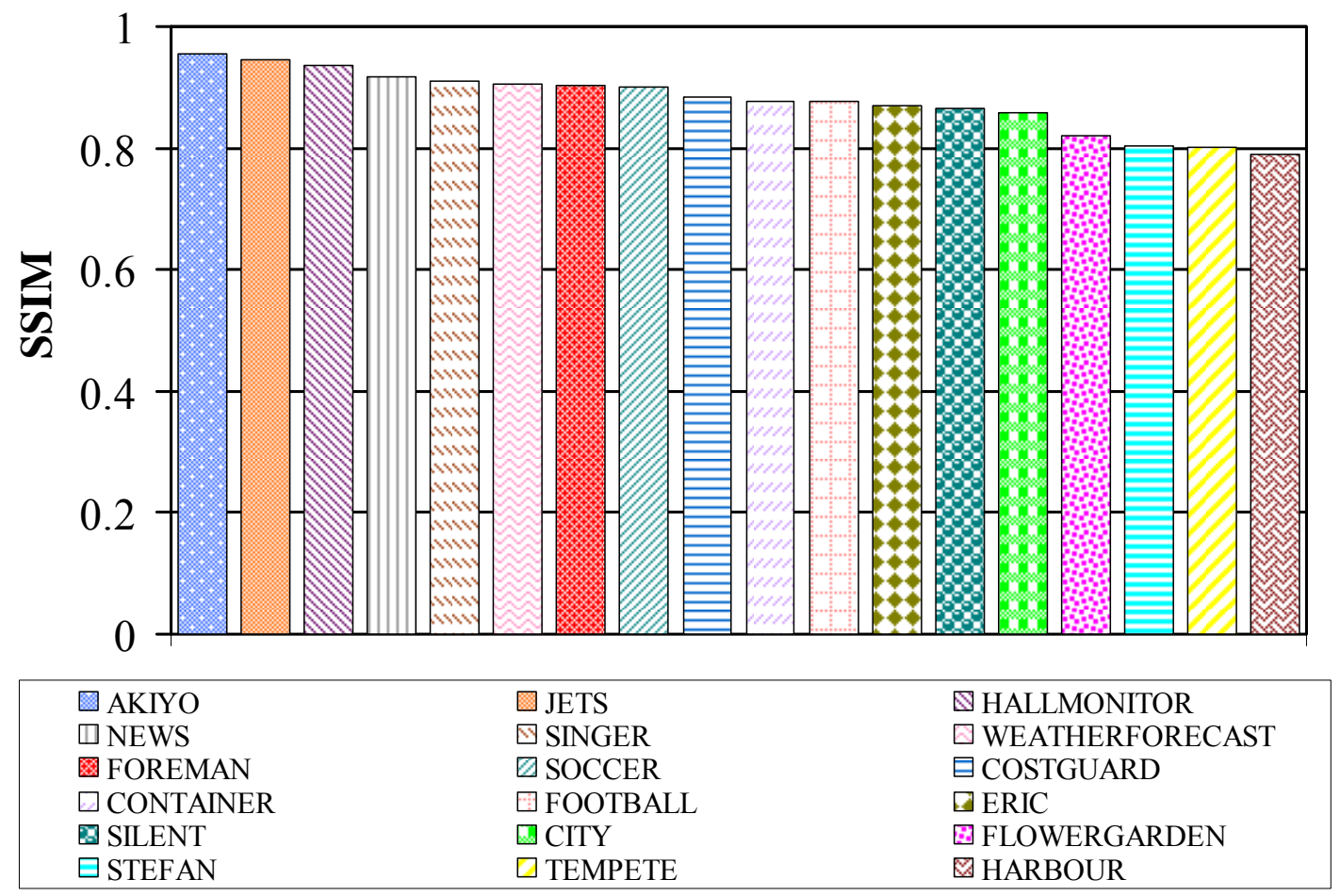

Fig. 4. The SSIM results of the down/up-sampling experiment (the first experiment)

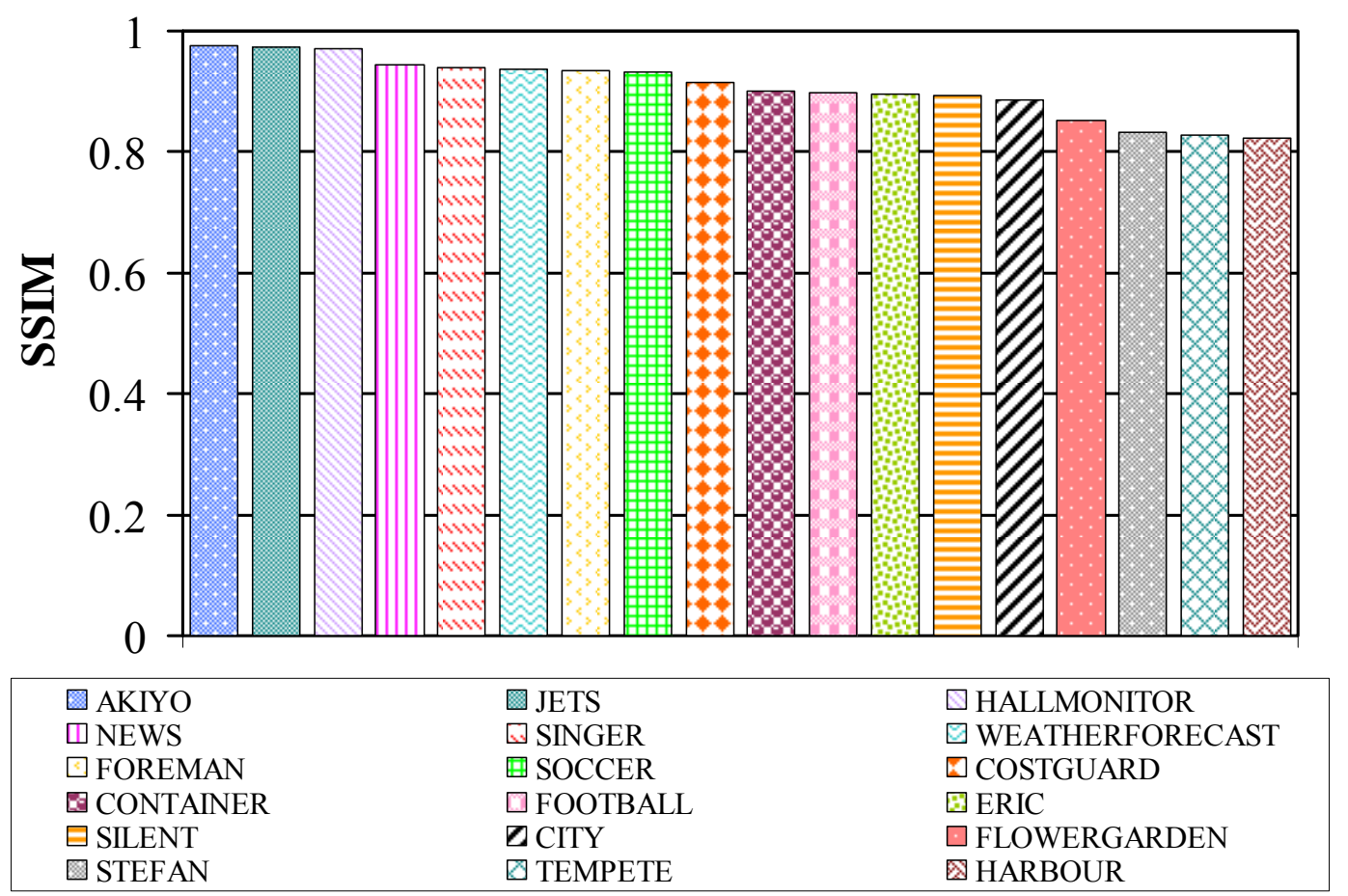

Fig. 5. The SSIM results of the coarsely quantized experiment (the second experiment)

In order to measure contour density, the number of pixels that are set to 1 is computed in every frame of a video sequence. The total is then normalized using the NoF and $S$ (i.e., spatial resolution) to provide consistency across different video sequences, as follows: 


$$
C=\frac{\sum_{i=1}^{\mathrm{NOF}} \delta(i)}{\mathrm{NoF} . S}
$$

where $C$ is the structural feature value of a video sequence, which is used for SFL classification. $\delta$ is the number of edge pixels in the $i^{\text {th }}$ frame of the sequence. $C$ was tested with a set of video sequences (i.e., 18 video sequences). Fig. 6 presents the $C$ results for the video sequences that have CIF resolution and $30 \mathrm{fps}$. In order to prove that (3) provides similar $C$ results for the derivation of a video sequence with different spatial resolutions and frame rates, the $C$ results of a selected sequence (i.e., Soccer) are also illustrated in Fig. 7.

In this work, the SFLs were split into two generic categories: low and highly complex. As shown in Table II, these classes are represented with X and Y, respectively. Similar to the ML classes, $K$-median clustering method [Mangasarian et. al., 2004] is used to ascertain the borders for each of the classes, which are also shown in Table II. A video sequence can be classified into one of these classes considering the borders of the $C$ values. Due to the cross matching of the MLs and SFLs presented in Tables I and III, respectively, the video sequences are classified into eight classes as shown in Table III.

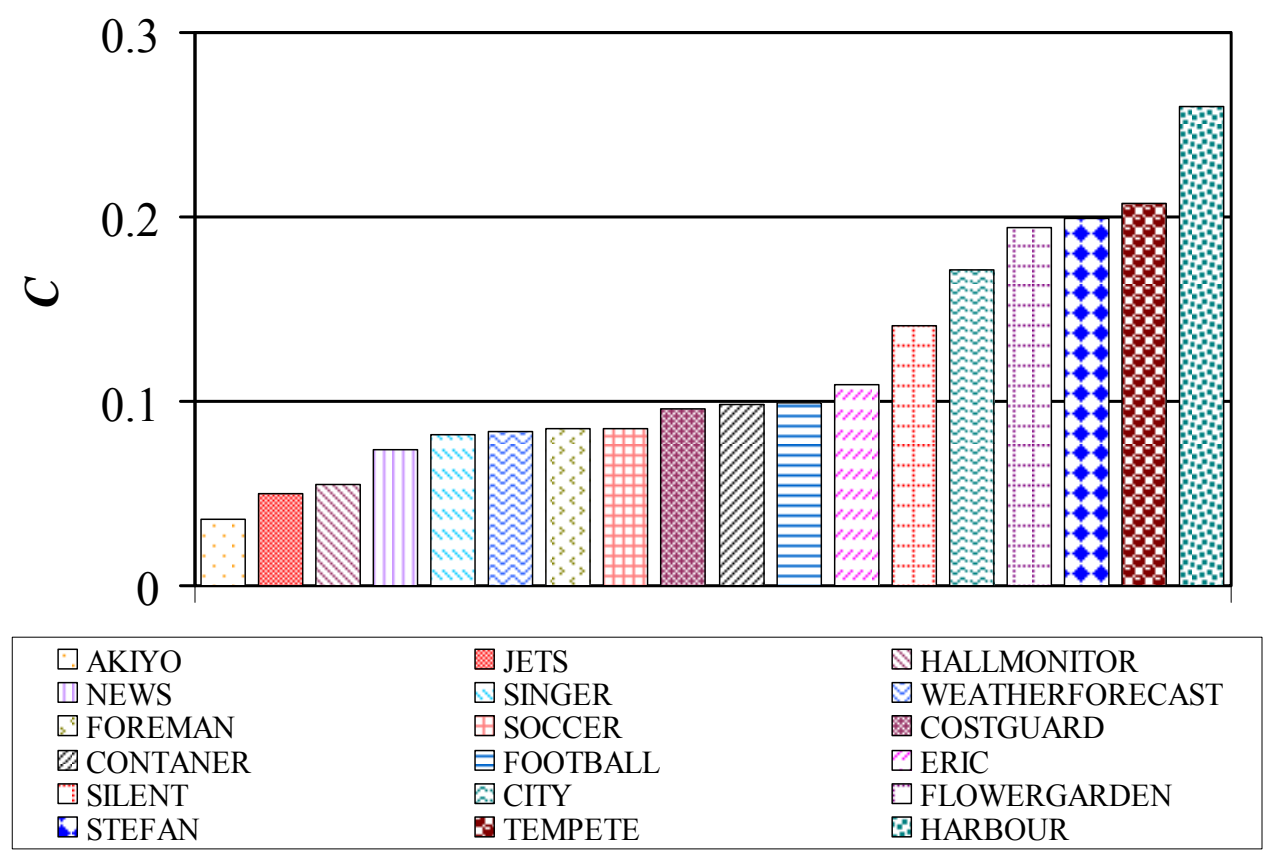

Fig. 6. The $C$ measurement results for different video sequences 


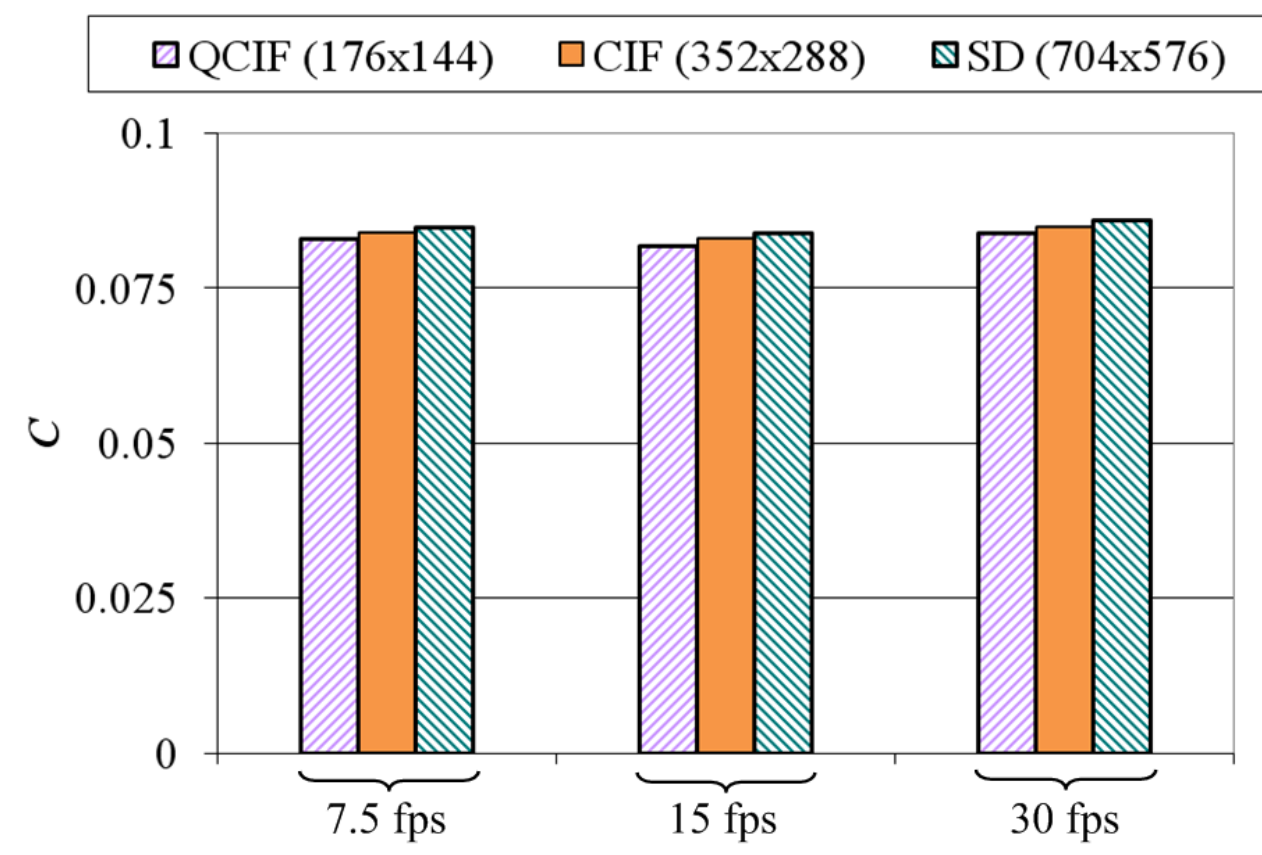

Fig. 7. The $C$ results for the Soccer sequence that has three different spatial resolutions (QCIF, CIF, and SD) and frame rates $(7.5,15$, and $30 \mathrm{fps})$.

TABLE II

THE STRUCTURAL FEATURE LEVELS

\begin{tabular}{|c|c|c|}
\hline SFL & Structural Feature & $C$ values \\
\hline $\mathrm{X}$ & Low & $0<C \leq 0.1$ \\
\hline $\mathrm{Y}$ & High & $C>0.1$ \\
\hline
\end{tabular}

TABLE III

Classes OF VIDEO SEQUENCES

\begin{tabular}{|c|c|c|c|c|c|c|c|c|}
\hline Class & 1 & 2 & 3 & 4 & 5 & 6 & 7 & 8 \\
\hline ML & $\mathrm{A}$ & $\mathrm{B}$ & $\mathrm{C}$ & $\mathrm{D}$ & $\mathrm{A}$ & $\mathrm{B}$ & $\mathrm{C}$ & $\mathrm{D}$ \\
\hline SFL & $\mathrm{X}$ & $\mathrm{X}$ & $\mathrm{X}$ & $\mathrm{X}$ & $\mathrm{Y}$ & $\mathrm{Y}$ & $\mathrm{Y}$ & $\mathrm{Y}$ \\
\hline
\end{tabular}

\section{UTILITY FUNCTION}

A generic UF, which defines the adaptation parameters (i.e., frame rates, spatial resolutions, and quality) and the weights specifying the relative importance of a parameter with respect to each other, is proposed in this paper. Utility is the measure of satisfaction or experience of users when a video sequence that has a specific spatial resolution, frame rate, and quality is viewed. Thus, the optimization of the UF presents the maximum user satisfaction in terms of frame rate, spatial resolution, and quality [Prangl et. al., 2007]. The general definition of the UF is: 


$$
U F=W_{F} F+W_{S} S+W_{Q} Q
$$

where, $F$ is the frame rate, $S$ is the spatial resolution, and $Q$ is the quality of a video sequence, which is the measure of quality degradation introduced to the video sequence in terms of distortion or artifacts. $W_{F}, W_{S}$, and $W_{Q}$ denote the weights that refer to how much influence the frame rate, spatial resolution, and quality parameters have on the UF, respectively. Thus, the UF is a weighted summation of $\mathrm{F}, \mathrm{S}$, and Q. In (4), $W_{F}+W_{S}+W_{Q}=1$, which means that there is a trade-off analysis between the weights of the frame rate, spatial resolution, and quality parameters while predicting the values of these parameters. For instance, when the $W_{F}$ is higher than $W_{S}$ and $W_{Q}$, the video with the highest available frame rate is predicted by the UF. It should be noted that the outcome of the UF is considered as unit-less. The quantitative values of the weights (i.e., $W_{F}, W_{S}$, and $W_{Q}$ ) depend on the ML and SFL of a video sequence. Accordingly, these weightings are represented as the functions of ML and SFL as follows:

$$
\begin{gathered}
W_{F}=f_{F}(\mathrm{ML}, \mathrm{SFL}) \\
W_{S}=f_{S}(\mathrm{ML}, \mathrm{SFL}) \\
W_{Q}=f_{Q}(\mathrm{ML}, \mathrm{SFL})
\end{gathered}
$$

In order to obtain $f_{F}(\mathrm{ML}, \mathrm{SFL}), f_{S}(\mathrm{ML}, \mathrm{SFL})$, and $f_{Q}(\mathrm{ML}, \mathrm{SFL})$ for the sequences that belong to different classes, firstly subjective tests are carried out. In this way, it can be clearly concluded that the subjective user experience is related to the UF. Secondly, the results of the subjective tests are incorporated into multiple regression analysis.

\section{SUBJECTIVE ASSESSMENTS}

Subjective quality assessment is an essential method to monitor users' preferences for video contents that have different properties [Yamazaki et. al., 2001]. The main aim of the subjective experiments is to evaluate the variations of the user satisfaction with respect to changing frame rate, spatial resolution, and quality of test video sequences under a constant bit rate restriction.

\subsection{Stimuli}

The test sequences used in subjective experiments were formed of eight video sequences, namely; Eric, Container, Jets, Soccer, Silent, Harbor, City, and Stefan. There are no benchmark video sequences hence the video sequences 
are selected based on their different motion and structural feature characteristics. Each sequence is a representation of the video sequence classes ranging from 1 to 8 (see Table III), respectively. These sequences contained the frames of the associated entire sequences ranging from $0-150,0-150,0-150,30-180,0-150,0-150,0-150$, and 10-160, respectively. Using a representative sequence for each class, a training phase was performed. Each of the test sequences were encoded with four different frame rates (i.e., 30, 15, 7.5, and 3.75), and three different spatial resolutions (i.e., SD, CIF, and QCIF). The three spatial resolutions were selected in such a way that they cover a representative range of mobile device display resolutions. The original-uncompressed versions of each video sequence were used as the references during the tests. Seven different channel bandwidth values (i.e., 96, 128, 192, $256,384,512$, and $768 \mathrm{kbps})$ were selected as the target bit rates. In order to match the bit rates of the encoded video sequences to the target source rates, different constant QP sets were utilized for scalable encoding of the base and spatial enhancement layers using JSVM 9.13.1 [JSVM 9.13.1 Software]. The stream that can be extracted from an encoded sequence is called as a scalable sub-stream in this paper. The sets producing sub-streams best matching with the target source rates were exploited to encode each sequence. Scalable sub-streams that have the QCIF, CIF, and SD resolutions at $3.75,7.5,15$, and 30 fps and target source rates were extracted from the encoded sequences and assessed by the viewers during the experiments.

\subsection{Test Methodology}

The Double Stimulus Impairment Scale (DSIS) methodology, as described in the ITU-R BT.500-11 [ITURecommendation BT.500-11, 2002], was used throughout the subjective evaluation tests. Thus, during the tests, the videos were shown in pairs: the first video was the reference, and the second video was the impaired one. The subjects were asked to assess the impaired video considering the associated reference. Subjective preferences were computed using Mean Opinion Score (MOS), which is the arithmetic mean of the individual scores given by the subjects [ITU-Recommendation BT.500-11, 2002]. An assessment scale ranging from 1 to 5 was used to rate the sequences. A score of 5 means the impaired video has the same perceptual quality as the reference one, while a score of 1 means it has high perceived impairment with respect to the reference.

\subsection{Test Procedure}

The tests were performed at the I-Lab of University of Surrey. A 23"-Dell display was used during the experiments 
to display the sequences. The resolution of the display is $1680 \times 1050$ pixels. For all of the experiments, the following procedure was applied. A short training session took place before the experiment commenced, so that the subjects could get acquainted with the test videos and the assessment scale. The video clips were displayed in their original sizes. A gray scale video was used to fill the gap for the remaining parts of the display. The viewing distance for the observers was not fixed. However, it was kept in compliance with the Preferred Viewing Distance (PVD) of the ITUR BT.500.11 [ITU-Recommendation BT.500-11, 2002]. During the tests, the video pairs were displayed randomly, so that the subjects were not prejudiced by a priori knowledge of presentation ordering. At the end of each pair, the subjects were given sufficient time to register their marks using the assessment scale described above.

\subsection{Viewers}

98 viewers (42 females and 56 males) participated in the experiments. Their ages ranged from 18 to 40, with an average of 27. None of the observers was previously involved in picture quality evaluations as part of their work, and they were not experienced assessors. They all reported normal and corrected eyesight. Owing to the large volume of the test sequences, the sequences were split into 12 groups, each consisted of 56 different video clips. 18 subjects assessed each group. Some of the subjects participated in more than one group. 2 observers were detected as outliers from each group using the outlier screening method discussed in [ITU-Recommendation BT.500-10, 2000]. Thus, in total 24 observers were detected as outliers, and the results of the remaining 74 viewers (33 females and 41 males) were used in the experiments. After the experiments, the MOSs and confidence intervals [ITU-Recommendation BT.500-11, 2002] were computed. After the outliers were eliminated, 16 subjects were used to calculate the MOSs in each group.

\section{EXPERIMENTAL RESULTS AND THEIR ANALYSIS}

In this section, the subjective test results are analyzed in order to specify the weights of the adaptation parameters (i.e., frame rate, spatial resolution, and quality) used in the UF (i.e., (4)). As the first step of the analysis, the frame rate, spatial resolution, and quality preferences of the viewers for the sub-streams of the test video sequences belonging to different ML and SFL classes, are discussed. To determine the frame rate and spatial resolution preferences of the viewers in this step, the average MOSs of the viewers are computed for the sub-streams that have the different spatial resolutions (i.e., QCIF, CIF, SD) or frame rates (i.e., 3.75, 7.5, 15, $30 \mathrm{fps}$ ) used in the experiments for each 
target bit rate. The average MOSs for all the sub-streams of each test video sequence is calculated to obtain the quality preferences of the viewers. As the second step of the analysis, the frame rate, spatial resolution, and quality preferences of the viewers are integrated together for each ML and SFL class. Following, the weighing factors table, which is produced using the integration results, is introduced.

\subsection{Frame Rate, Spatial Resolution, and Quality Preferences of the Viewers for the Test Video Sequences Belonging} to Different ML and SFL Classes

In this section, the frame rate, spatial resolution, and quality preferences of the viewers are illustrated using the graphs of four selected test sequences (i.e., Eric, Silent, City, and Stefan), which belong to Classes 1, 5, 7, and 8 (see Table III), respectively.

\subsubsection{Frame Rate Preferences}

Fig. 8 presents the MOS versus bit rate plot showing the users' preferences for the Eric sub-streams at different frame rates. As can be observed from the figure, the Eric sub-streams that have a frame rate of $3.75 \mathrm{fps}$ are rated with the highest MOSs by the viewers at bit rates between 96-192 kbps. The reason for this is that the lower the frame rate, the higher the number of bits allocated per frame of the video sequence at a target bit rate. Thus, high frame quality is achieved when the Eric sub-streams have a 3.75 fps frame rate at these low bit rates (i.e., 96-192 kbps). Even though the frame rate of the sub-streams is low, the high frame quality enhances the video perception of users at these low bit rates. Thus, the frame rate is less of an issue with the Eric sequence due to the low motion activity presented in it. As can be realized from the figure, the frame rate preferences of the viewers shift from lower to higher frame rates (i.e., 3.75 to 7.5 and 7.5 to $15 \mathrm{fps}$ ) at 256 and $512 \mathrm{kbps}$ bit rates, respectively. The reason behind these shifts is that the perceived video quality saturates at lower frame rates due to high frame quality, and it is then enhanced with higher frame rates (e.g., 256 and $512 \mathrm{kbps}$ bit rates). 


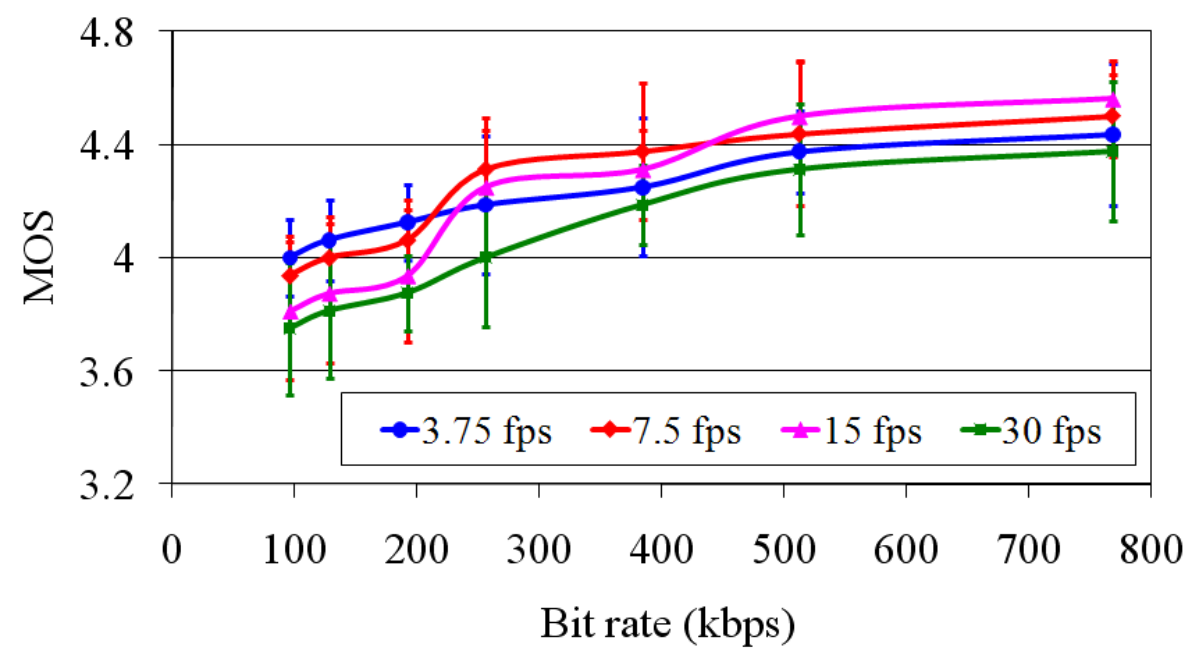

Fig. 8. The Eric sequence MOS versus bit rate results based on various frame rates

Fig. 9 presents the frame rate preferences of the viewers with regard to the Silent sequence. As seen from the figure, 3.75 fps has the highest MOSs between 96 and 256 kbps bit rates. From 256 kbps onwards, the sub-streams with 7.5 fps are preferred until a bit rate of $512 \mathrm{kbps}$ is reached, and the sub-streams with 15 fps have the highest MOSs after that. The Silent and Eric sequences present similar motion activities. However, the bit rates at which the frame rate choices start varying (i.e., from 3.75 to 7.5 and from 7.5 to $15 \mathrm{fps}$ ) are higher for the Silent sequence compared to those of the Eric sequence. The reason is that the Silent sequence has higher complex structural feature than the Eric sequence. When a video sequence presents higher complex structural feature, the details in the frames of the sequence requires a greater number of bits as a video sequence with a low complex feature to produce similar video perception when both sequences have same the frame rates.

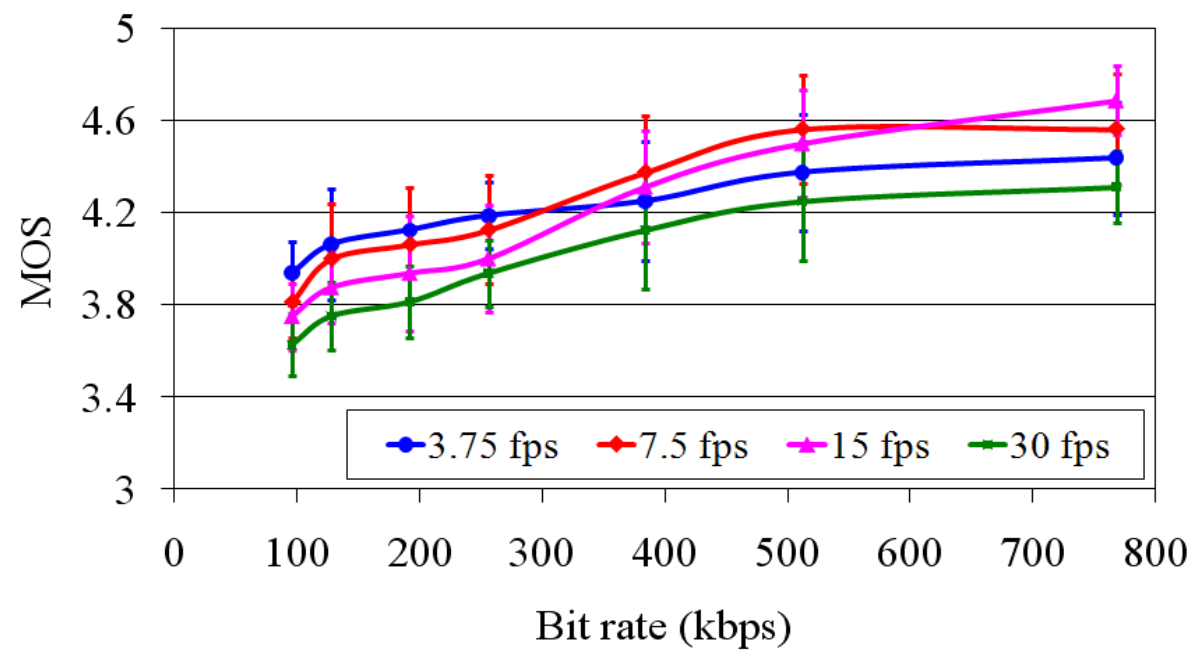

Fig. 9. The Silent sequence MOS versus bit rate results based on various frame rates 
Fig. 10 shows the MOS versus bit rate graph presenting the users' preferences for the City sub-streams with different frame rates. As seen from the figure, the City sub-streams that have $15 \mathrm{fps}$ are preferred by the viewers compared to those that have $3.75,7.5$, or $30 \mathrm{fps}$ until a bit rate of $256 \mathrm{kbps}$ is reached. As can also be observed from the figure, the City sub-streams that have 30 fps are preferred by the users when the bit rate is between 384 and 768 kbps. Even though, frame quality is higher for a video sequence at a low frame rate (i.e., 3.75 and $7.5 \mathrm{fps}$ ) compared to that at a high frame rate (i.e., 15 and $30 \mathrm{fps}$ ), the perceptual video quality is improved with increasing frame rates for the City sequence, which has a medium-high motion activity. The reason is that when the motion activity in a video sequence is medium-high or high, high frame rates represent the real-world active scenes more naturally.

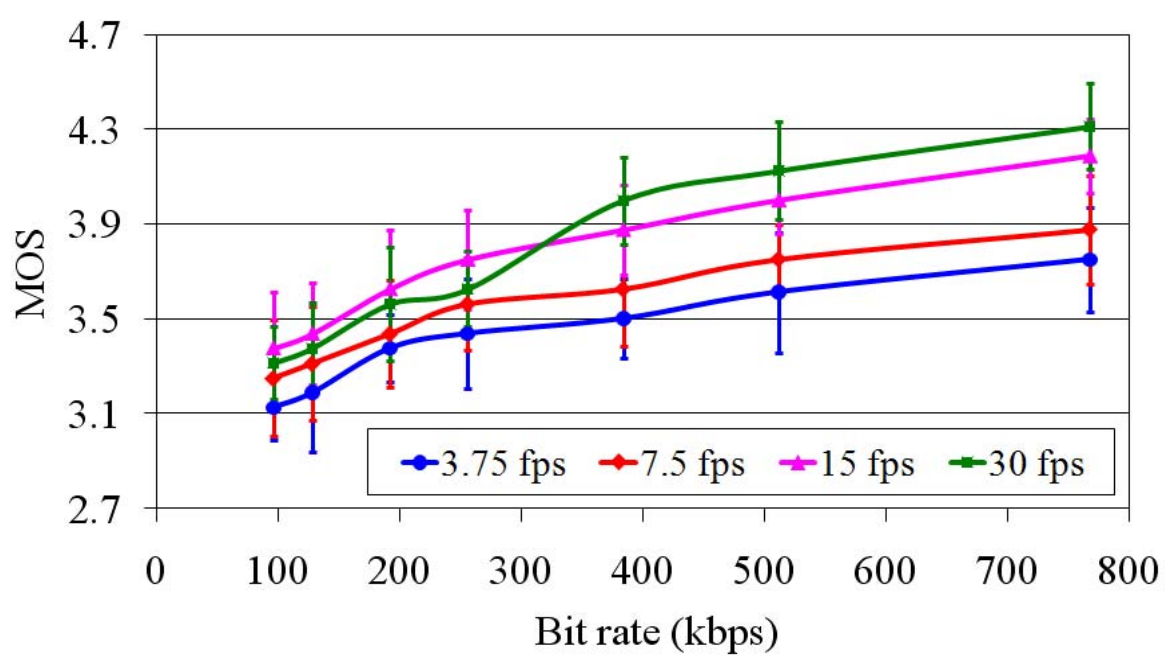

Fig. 10. The City sequence MOS versus bit rate results based on various frame rates

The Stefan sequence MOS versus bit rate results based on various frame rates can be seen in Fig. 11. As can be realized from the figure, the subjects prefer the sub-streams with high frame rates (i.e., 15 and $30 \mathrm{fps}$ ) for the Stefan sequence in a similar manner to the City sequence. Nevertheless, the Stefan sub-streams with 30 fps start achieving higher MOSs at lower bit rates compared to the City sub-streams. The reason behind this is that the Stefan sequence presents higher motion activity compared to the City sequence. 


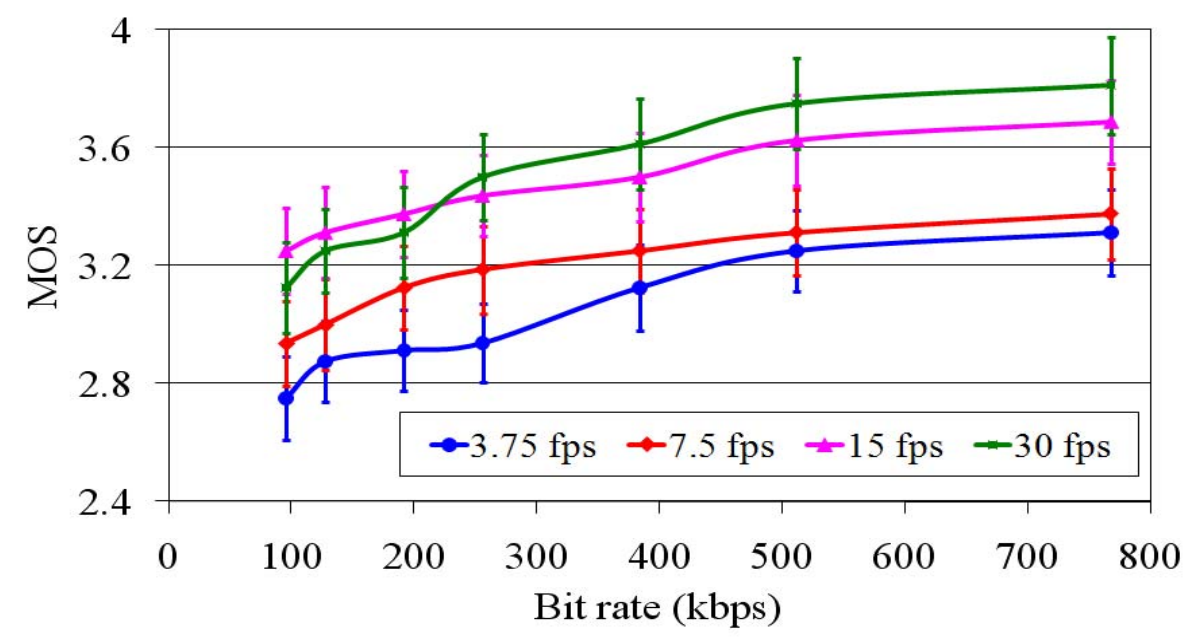

Fig. 11. The Stefan sequence MOS versus bit rate results based on various frame rates

The findings observed for the relationships between the frame rate preferences of the viewers and the motion and structural features of video sequences are summarized in Table IV and the bullet points presented following the table.

TABLE IV

THE FRAME RATES PREFERRED BY THE VIEWERS FOR THE TEST VIDEO SEQUENCES

\begin{tabular}{|c|c|c|c|c|c|c|c|c|c|}
\hline \multicolumn{2}{|c|}{ Classes } & 1 & 2 & 3 & 4 & 5 & 6 & 7 & 8 \\
\hline \multicolumn{2}{|c|}{ Motion Activity } & Low & $\begin{array}{c}\text { Low- } \\
\text { Medium }\end{array}$ & $\begin{array}{l}\text { Medium- } \\
\text { High }\end{array}$ & High & Low & $\begin{array}{c}\text { Low- } \\
\text { Medium }\end{array}$ & $\begin{array}{l}\text { Medium- } \\
\text { High }\end{array}$ & High \\
\hline \multicolumn{2}{|c|}{ Structural Feature } & Low & Low & Low & Low & High & High & High & High \\
\hline \multicolumn{2}{|c|}{ Test Sequence } & Eric & Container & Jets & Soccer & Silent & Harbor & City & Stefan \\
\hline \multirow{7}{*}{ 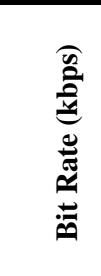 } & 96 & $3.75 \mathrm{fps}$ & $3.75 \mathrm{fps}$ & $15 \mathrm{fps}$ & $15 \mathrm{fps}$ & $3.75 \mathrm{fps}$ & $3.75 \mathrm{fps}$ & $15 \mathrm{fps}$ & $15 \mathrm{fps}$ \\
\hline & 128 & $3.75 \mathrm{fps}$ & $3.75 \mathrm{fps}$ & $15 \mathrm{fps}$ & $15 \mathrm{fps}$ & $3.75 \mathrm{fps}$ & $3.75 \mathrm{fps}$ & $15 \mathrm{fps}$ & $15 \mathrm{fps}$ \\
\hline & 192 & $3.75 \mathrm{fps}$ & $7.5 \mathrm{fps}$ & $15 \mathrm{fps}$ & $30 \mathrm{fps}$ & $3.75 \mathrm{fps}$ & $3.75 \mathrm{fps}$ & $15 \mathrm{fps}$ & $15 \mathrm{fps}$ \\
\hline & 256 & $7.5 \mathrm{fps}$ & $7.5 \mathrm{fps}$ & $30 \mathrm{fps}$ & $30 \mathrm{fps}$ & $3.75 \mathrm{fps}$ & $7.5 \mathrm{fps}$ & $15 \mathrm{fps}$ & $30 \mathrm{fps}$ \\
\hline & 384 & $7.5 \mathrm{fps}$ & $15 \mathrm{fps}$ & $30 \mathrm{fps}$ & $30 \mathrm{fps}$ & $7.5 \mathrm{fps}$ & $7.5 \mathrm{fps}$ & $30 \mathrm{fps}$ & $30 \mathrm{fps}$ \\
\hline & 512 & $15 \mathrm{fps}$ & $15 \mathrm{fps}$ & $30 \mathrm{fps}$ & $30 \mathrm{fps}$ & $7.5 \mathrm{fps}$ & $15 \mathrm{fps}$ & $30 \mathrm{fps}$ & $30 \mathrm{fps}$ \\
\hline & 768 & $15 \mathrm{fps}$ & $15 \mathrm{fps}$ & $30 \mathrm{fps}$ & $30 \mathrm{fps}$ & $15 \mathrm{fps}$ & $15 \mathrm{fps}$ & $30 \mathrm{fps}$ & $30 \mathrm{fps}$ \\
\hline
\end{tabular}

The summary of the observations for the frame rate preferences of the viewers:

- The lower the frame rate, the higher the frame quality of a video sequence is for a given bit rate.

- When a video sequence has high motion activity, the viewers prefer the video sequence to have a high frame rate since it presents highly active real-world scenes more naturally.

- Fewer bits are required for a video sequence, which shows structural features of low complexity in order to provide a similar video perception level as a sequence at the same frame rate, but showing more complex structural features. 


\subsubsection{Spatial Resolution Preferences}

For the Eric sequence, the MOS versus bit rate results for various spatial resolutions are shown in Fig. 12. As seen from the figure, the CIF resolution is rated with the highest MOSs between 96 and $128 \mathrm{kbps}$. The reason is that the lower the spatial resolution the higher the frame quality is at a target bit rate. The CIF resolution is not the lowest resolution used in the subjective experiments however the Eric sub-streams with CIF resolution do not require a large amount of bits to improve the video perception due to the low motion and low complex structural feature characteristics presented in this sequence. As can also be observed from the figure, when the video sequences are encoded at bit rates higher than $128 \mathrm{kbps}$, frame quality of the Eric sub-streams that have higher spatial resolution (i.e., the SD resolution) starts increasing the perceived video quality. Therefore, the viewers give the highest MOSs to the Eric substreams with SD resolution between 192 and 768 kbps.

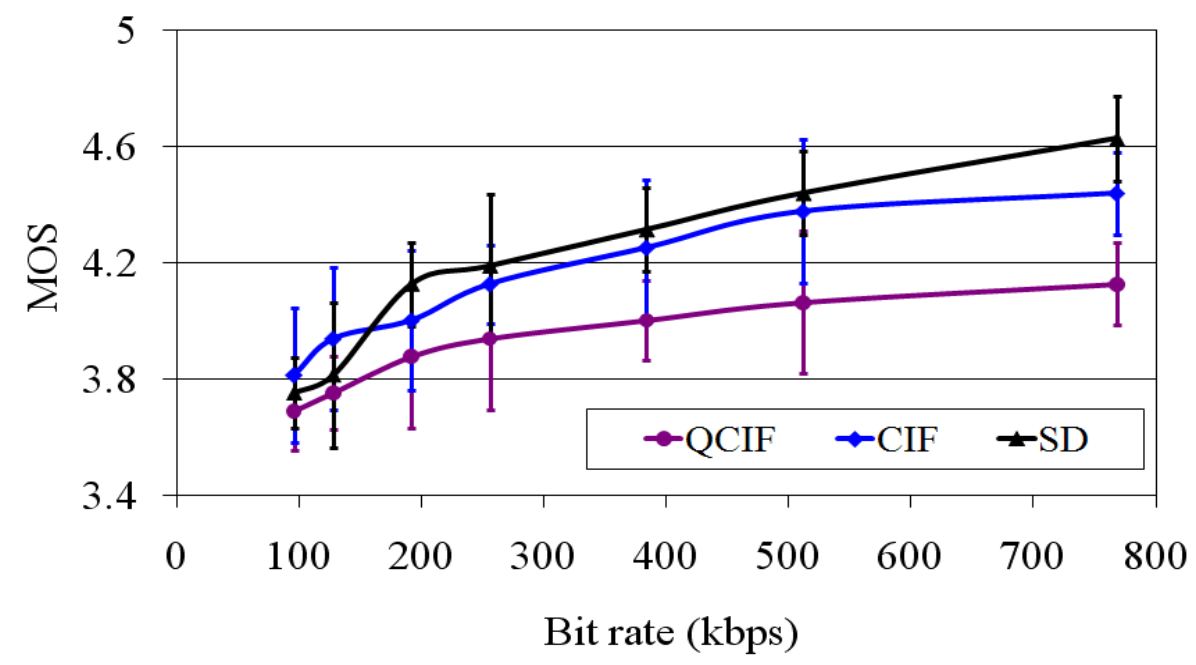

Fig. 12. The Eric sequence MOS versus bit rate results based on various spatial resolutions

The spatial resolution preferences of the subjects for the Silent sequence encoded at 96-768 kbps are illustrated in Fig. 13. Similar to the Eric sequence, which contains low motion and low complex structural features, the Silent substreams with CIF resolution are rated with the highest MOSs by the viewers at low bit rate(s) (i.e., $96 \mathrm{kbps})$ while the SD resolution receives the highest MOSs from the viewers at medium and high bit rates (i.e., 128-768 kbps). However, the medium and high bit rate ranges, in which the Silent sub-streams having SD resolutions are preferred, are wider compared to those of the Eric sub-streams. This is due to the greater complexity of the structural feature presented in the Silent sequence compared to that in the Eric sequence. The higher the spatial resolution of a video sequence with a high complex structural feature, the better the structural details in the frames of the sequence is visual- 
ly distinguishable. Thus, the Silent sub-streams with spatial resolution are preferred by the viewers at these particular bit rates.

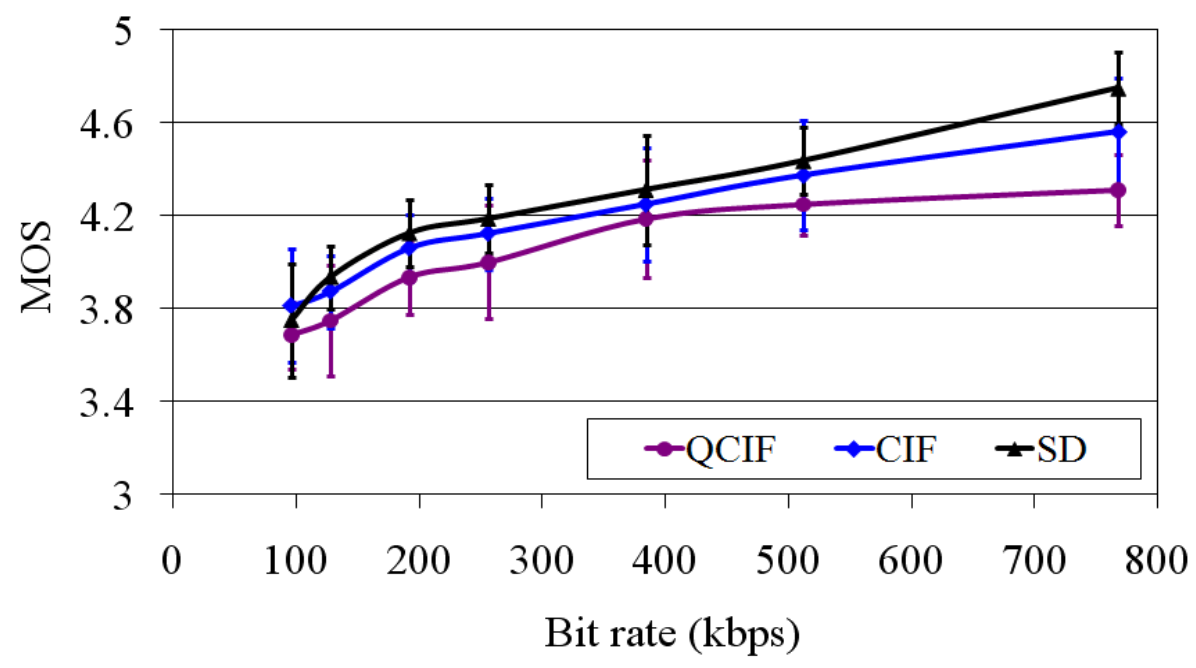

Fig. 13. The Silent sequence MOS versus bit rate results based on various spatial resolutions

Fig. 14 illustrates the spatial resolution preferences of the viewers for the City sub-streams that have different bit rates. As seen from the figure, the City sub-streams with QCIF, CIF, and SD resolutions are preferable at low (i.e., 96 kbps), medium (i.e., 128-256 kbps), and high (i.e., 384-768 kbps) bit rates, respectively. When the structural feature of a low or low-medium motion video sequence is highly complex (e.g., the Silent sequence), the viewers prefer to view the sequence at high spatial resolution regardless of the bit rate. However, the viewers prefer to view the City sequence at low spatial resolutions (i.e., QCIF) at a low bit rate (i.e., $96 \mathrm{kbps}$ ). When the bit rate increases, the CIF and SD resolutions are preferred by the viewers for the City sequence. The reason of these observations is that when the motion activity of a video sequence is medium-high, the video perception can only be improved using the substreams with low spatial resolution, in which the frame quality is high at low bit rates (e.g., $96 \mathrm{kbps})$. When the bit rate increases, the perceived video quality of the sub-streams that have higher spatial resolutions starts to increase. 


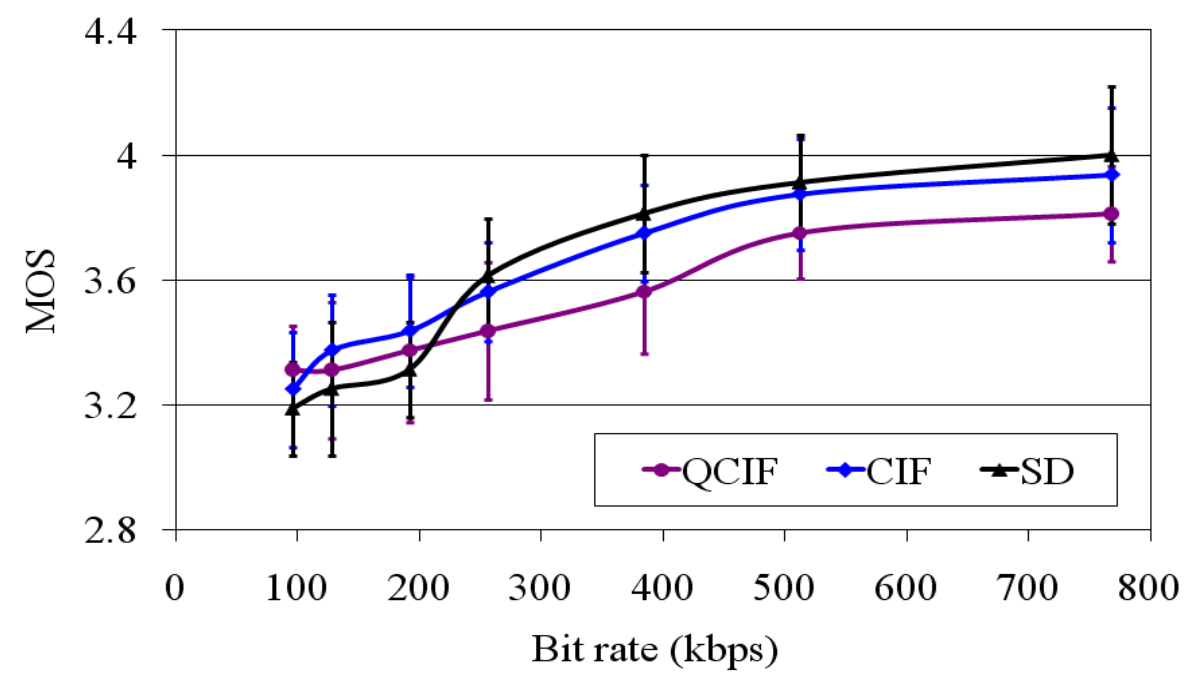

Fig. 14. The City sequence MOS versus bit rate results based on various spatial resolutions

As can be seen from Fig. 15, which shows the spatial resolution preference of the users for the Stefan sub-streams that have different spatial resolutions, the subjects are more inclined to select QCIF resolution at a low bit rate range (i.e., 96 to $128 \mathrm{kbps}$ ). Nevertheless, this preference changes to CIF resolution between 192 and $256 \mathrm{kbps}$. At bit rates above $256 \mathrm{kbps}$, the Stefan sub-streams that have SD spatial resolution receive the highest MOS from the viewers.

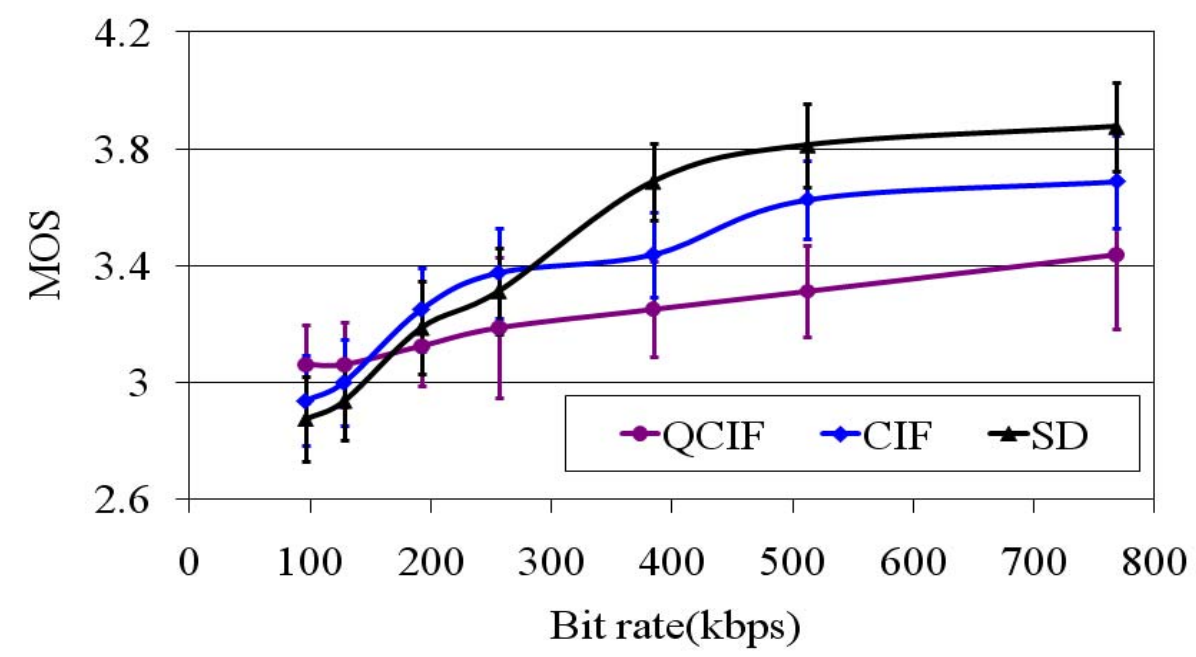

Fig. 15. The Stefan sequence MOS versus bit rate results based on various spatial resolutions

The observations about the relationships between the spatial resolution preferences of the viewers and the motion and structural features of video sequences are summarized in Table $\mathrm{V}$ and the bullet points presented after the table. 
TABLE V

THE SPATIAL RESOLUTIONS PREFERRED BY THE VIEWERS FOR THE TEST VIDEO SEQUENCES

\begin{tabular}{|c|c|c|c|c|c|c|c|c|c|}
\hline \multicolumn{2}{|c|}{ Classes } & 1 & 2 & 3 & 4 & 5 & 6 & 7 & 8 \\
\hline \multicolumn{2}{|c|}{ Motion Activity } & Low & $\begin{array}{c}\text { Low- } \\
\text { Medium }\end{array}$ & $\begin{array}{l}\text { Medium- } \\
\text { High }\end{array}$ & High & Low & $\begin{array}{c}\text { Low- } \\
\text { Medium }\end{array}$ & $\begin{array}{l}\text { Medium- } \\
\text { High }\end{array}$ & High \\
\hline \multicolumn{2}{|c|}{ Structural Feature } & Low & Low & Low & Low & High & High & High & High \\
\hline \multicolumn{2}{|c|}{ Test Sequence } & Eric & Container & Jets & Soccer & Silent & Harbor & City & Stefan \\
\hline \multirow{7}{*}{ 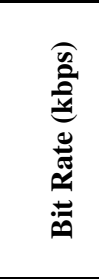 } & 96 & CIF & CIF & QCIF & QCIF & CIF & CIF & QCIF & QCIF \\
\hline & 128 & CIF & CIF & $\mathrm{CIF}$ & QCIF & SD & CIF & CIF & QCIF \\
\hline & 192 & SD & $\mathrm{CIF}$ & $\mathrm{CIF}$ & $\mathrm{CIF}$ & SD & $\mathrm{SD}$ & $\mathrm{CIF}$ & $\mathrm{CIF}$ \\
\hline & 256 & SD & SD & $\mathrm{CIF}$ & $\mathrm{CIF}$ & SD & SD & SD & CIF \\
\hline & 384 & SD & SD & SD & $\mathrm{CIF}$ & SD & SD & SD & SD \\
\hline & 512 & SD & SD & SD & SD & SD & SD & SD & SD \\
\hline & 768 & SD & SD & SD & SD & SD & SD & SD & SD \\
\hline
\end{tabular}

The summary of the observations for the spatial resolution preferences of the viewers:

- The higher the motion activity of a video sequence, the higher the bit rate required to achieve a similar video perception level with a video sequence that has lower motion activity but similar spatial resolution.

- When a low or low-medium motion video sequence presents a high complex structural feature, the higher the spatial resolution, the better the video perception at low bit rates.

- The lower the spatial resolution, the higher the frame quality is for medium-high and high motion video sequences at low bit rates.

\subsubsection{Quality Preferences}

Fig. 16 illustrates the average MOS and VQM results for the sub-streams of the test video sequences encoded in bit rates from 96 to $768 \mathrm{kbps}$. In order to provide consistency with the MOS results, a score of 5 in the VQM grades means the impaired video has no video quality degradations compared to the reference, while a VQM grade of 1 represents high video quality degradations in this paper. From the graphs, it is observed that both the average MOS and VQM grades gradually increase when going from low to high bit rates. As can also be seen from the graphs, the MOS curves are always below the VQM curves. The reason behind this is that the video sequences used as references to compute the VQM grades of the sub-streams had similar spatial resolutions and frame rates to the associated sub-streams. However, during the subjective experiments, original-uncompressed video sequences, which had the highest spatial resolution, frame rate, and quality, were used as reference video sequences. Thus, the viewers assessed the sub-streams with respect to the video sequences that presented the best possible perceived video quality (i.e., original-uncompressed video sequences). Due to this reason, the average MOSs have lower values than the average VQM grades. It should be noted that human observers, who are the "true assessors" of video quality perception, par- 
ticipated in the subjective experiments. Therefore, it is expected that the observers provided the most reliable video quality assessments [Yamazaki et. al., 2001].

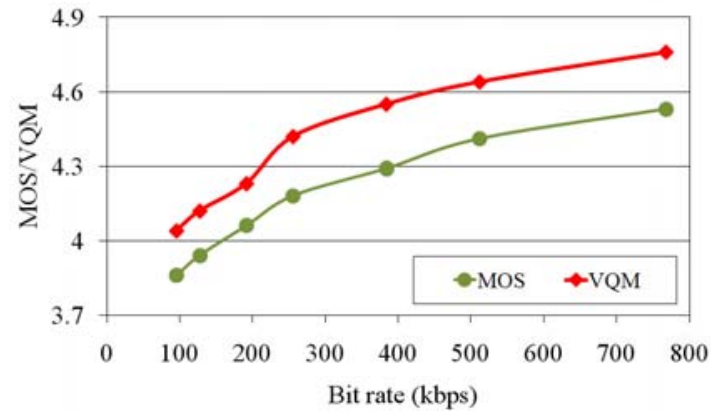

(a)

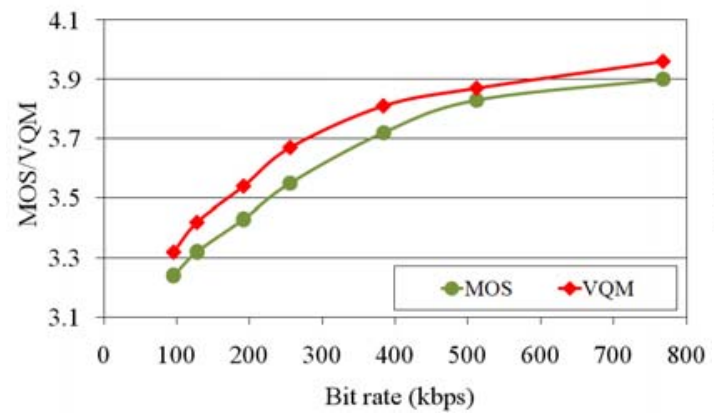

(c)

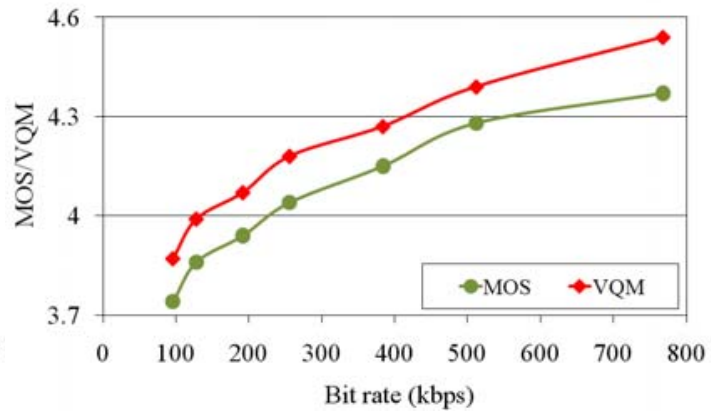

(b)

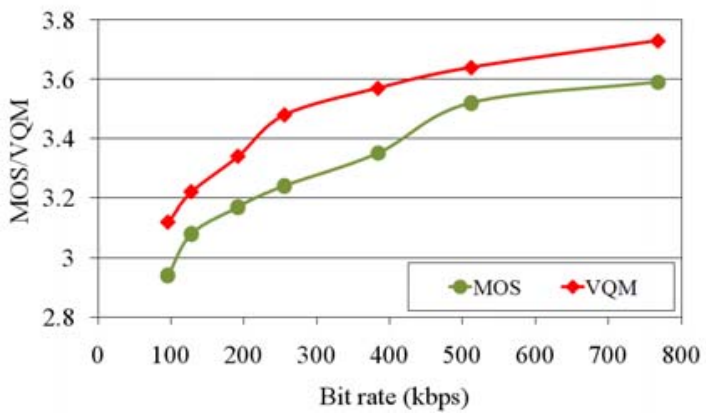

(d)

Fig. 16. The MOS and VQM versus bit rate results for the (a) Eric (b) Silent (c) City (d) Stefan test video sequences

\subsection{Integration of the Frame Rate, Spatial Resolution, and Quality Preferences of the Viewers for Each ML and SFL}

Class

As the second step in the analysis, a multiplicative integration scheme is required to integrate the viewers' preferences for the frame rate, spatial resolution, and quality of video sequences obtained through subjective experiments to determine the weights of the UF. Therefore, a multiple regression analysis [Devore, 1995] was performed using the results of the subjective experiments to determine the impacts of the frame rate, spatial resolution, and quality factors on the UF.

Regression equation models represent the dependent variables as functions of the independent variables. Here, the dependent variable is the UF, and the independent variables are the frame rate, spatial resolution, and quality. Accordingly, the general multiple regression equation is expressed as:

$$
U F^{\prime}=\widehat{W}_{F} \widehat{F}+\widehat{W}_{S} \widehat{S}+\widehat{W}_{Q} \widehat{Q}
$$

where $\hat{F}, \hat{S}$, and $\hat{Q}$ represent the predictor variables of frame rate, spatial resolution, and quality, respectively. 
$\widehat{W}_{F}, \widehat{W}_{S}$, and $\widehat{W}_{Q}$ are the regression coefficients of the unknown parameters, $W_{F}^{\prime}, W_{S}^{\prime}$, and $W_{Q}^{\prime}$. The objective here is to estimate the unknown parameters and then map the estimated values to the regression coefficients in (8). In order to avoid confusion between the parameters of the proposed UF equation in (4) and the multiple regression equation in (8), $U F, W_{F}, W_{S}$, and $W_{Q}$ in (4) are denoted as $U F^{\prime}, \hat{W}_{F}, \hat{W}_{s}$, and $\hat{W}_{Q}$ in (8), respectively.

The results obtained from the subjective experiments were separated into 84 sets that have four components each: $\left(F_{1}, S_{1}, Q_{1}, M O S_{1}\right),\left(F_{2}, S_{2}, Q_{2}, M O S_{2}\right), \ldots,\left(F_{84}, S_{84}, Q_{84}, M O S_{84}\right)$. Here, $F, S, Q$, and $M O S$ represent the frame rate, spatial resolution, quality values, and the average MOS calculated following the experiments, respectively. The subscripts of the $F, S, Q$, and $M O S$ represent the sequence number in the sets. 84 sets were prepared since the combination of four frame rates (i.e., 3.75, 7.5, 15, and $30 \mathrm{fps}$ ), three spatial resolutions (i.e., QCIF, CIF, and SD), and seven average quality values associated with seven bit rates (i.e., 96, 128, 192, 256, 384, 512, and $768 \mathrm{kbps}$ ) used during the subjective tests for each video sequence type was $4 \times 3 \times 7=84$. These sets were prepared for every video sequence type separately. In order to estimate the regression coefficients in (8), the following equation is formulated based on the multiple regression analysis [Hands et. al., 2004, Devore, 1995] firstly:

$$
f\left(W_{F}^{\prime}+W_{S}^{\prime}+W_{Q}^{\prime}\right)=\sum_{j}\left[M O S_{j}-\left(W_{F}^{\prime} F_{j}+W_{S}^{\prime} S_{j}+W_{Q}^{\prime} Q_{j}\right)\right]^{2}
$$

where $j$ represents the number of sets each of which have four components (i.e., $F, S, Q$, and $M O S$ ). Thereafter, the partial derivative of $f$ is taken with respect to $W_{F}^{\prime}, W_{S}^{\prime}$, and $W_{Q}^{\prime}$. Then, all of the partial derivatives are equated to zero to yield the equations below:

$$
\begin{gathered}
W_{F}^{\prime} \sum_{j=1}^{84} F_{j}+W_{S}^{\prime} \sum_{j=1}^{84} S_{j}+W_{Q}^{\prime} \sum_{j=1}^{84} Q_{j}=\sum_{j=1}^{84} M O S_{j} \\
W_{F}^{\prime} \sum_{j=1}^{84}\left(F_{j}\right)^{2}+W_{S}^{\prime} \sum_{j=1}^{84} S_{j} F_{j}+W_{Q}^{\prime} \sum_{j=1}^{84} Q_{j} F_{j}=\sum_{j=1}^{84} M O S_{j} F_{j} \\
\left.W_{F}^{\prime} \sum_{j=1}^{84} F_{j} S_{j}+W_{S}^{\prime} \sum_{j=1}^{84} S_{j}\right)^{2}+W_{Q}^{\prime} \sum_{j=1}^{84} Q_{j} S_{j}=\sum_{j=1}^{84} M O S_{j} S_{j} \\
W_{F}^{\prime} \sum_{j=1}^{84} F_{j} Q_{j}+W_{S}^{\prime} \sum_{j=1}^{84} S_{j} Q_{j}+W_{Q}^{\prime} \sum_{j=1}^{84}\left(Q_{j}\right)^{2}=\sum_{j=1}^{84} M O S_{j} Q_{j}
\end{gathered}
$$

These equations are solved by computing all of the constants related to $F, S$, and $Q$, and then using the Gaussian function [Douglas, 1971] as the elimination technique to determine the unknown parameters (i.e., $W_{F}^{\prime}, W_{S}^{\prime}$, and $W_{Q}^{\prime}$ ). Once these parameters were found, they were mapped to the regression coefficients (i.e., $\widehat{W}_{F}$, $\widehat{W}_{S}$, and $\widehat{W}_{Q}$ ). 
The results of the multiple regression analysis were then matched to the weights in the UF (i.e., (1)) for every sequence type. These weights are illustrated in Table VI.

The UF weights in the table reflect the priorities of the subjects' preferences regarding the scalability parameters. The results of the multiple regression analysis performed on the low motion and low-medium structural feature (i.e., Class 1) video sequence show that high spatial resolutions (i.e., $\left.W_{S}=0.40\right)$ are preferred by the subjects over high quality (i.e., $W_{Q}=0.37$ ). Nevertheless, the preference for the high frame rate is less than that for the high quality (i.e., $W_{F}=0.23$ ). Both high spatial resolution (i.e., $W_{S}=0.36$ ) and quality (i.e., $W_{Q}=0.33$ ) are preferred by the subjects for low-medium motion and low complex structural feature (i.e., Class 2) sequences. However, the preference for high frame rate is lower (i.e., $W_{F}=0.31$ ) than that for high spatial resolution and quality. The dominant factor that gets high subjective preference is the frame rate (i.e., $W_{F}=0.43$ ) for the medium-high motion, low structural feature (i.e., Class 3) video sequences. Spatial resolution (i.e., $W_{S}=0.31$ ) and quality (i.e., $W_{Q}=0.26$ ) follow this preference, respectively. For the high motion and low structural feature video sequences (i.e., Class 4), subjects are also inclined to make frame rate (i.e., $W_{F}=0.46$ ) based preferences over spatial resolution (i.e., $W_{S}=0.30$ ) and quality (i.e., $W_{Q}=$ $0.24)$ preferences. These findings of the low complex structural feature video sequences are also applicable for the high complex structural feature video sequences with respect to the MLs. However, the difference between the $W_{S}$ and $W_{Q}$ are higher for the high complex structural feature video sequences than those for the low complex structural feature video sequences. In other words, spatial resolution affects users' preferences more for the high complex structural feature video sequences compared to the low structural feature ones. From all of these results, it can be concluded that the amount of contributions of the scalable stream parameters (i.e., frame rate, spatial resolution, and quality) to the subjective preferences strongly depend on the type of a video content.

TABLE VI

THE WEIGHING FACTORS TABLE

\begin{tabular}{|c|c|c|c|}
\hline \multirow{2}{*}{ ML and SFL Class } & \multicolumn{3}{|c|}{ UF Weights } \\
\cline { 2 - 4 } & $W_{F}$ & $W_{S}$ & $W_{O}$ \\
\hline 1 & 0.23 & 0.40 & 0.37 \\
\hline 2 & 0.31 & 0.36 & 0.33 \\
\hline 3 & 0.43 & 0.31 & 0.26 \\
\hline 4 & 0.46 & 0.30 & 0.24 \\
\hline 5 & 0.22 & 0.43 & 0.35 \\
\hline 6 & 0.30 & 0.39 & 0.31 \\
\hline 7 & 0.42 & 0.35 & 0.23 \\
\hline 8 & 0.45 & 0.34 & 0.21 \\
\hline
\end{tabular}




\section{PROPOSED VIDEO ADAPTATION FRAMEWORK}

The proposed video adaptation framework is illustrated in Fig. 17. As seen from the figure, the framework is divided into three main modules namely; AQoS, adaptation, and UCD. The adaptation module, which can be placed in a network node, is the heart of this framework. The adaptation module includes Adaptation Decision Taking Engine (ADTE) and Adaptation Engine (AE) sub-modules to execute adaptation processes. As realized from the figure, the ADTE sub-module receives AQoS and UCD to make appropriate adaptation decisions in the form of adaptation parameters by solving the optimization problem, which was described in Section 6. AQoS supplies content-related metadata and UCD represents constraints specifications. In this way, the ADTE sub-module decides on the scalability parameters that can be sacrificed to fulfill the constraints, which are provided by UCD, using the content-related metadata provided by AQoS.

As seen from the AQoS module in the figure, the content related metadata described in AQoS includes scalable substreams' parameters (i.e., spatial resolution, frame rate, and quality), the VQM results of the scalable sub-streams, and the modeled UF. The ML and SFL classification blocks in the framework implement the ML and SFL classification algorithms. Additionally, the UF generator block of the framework includes the modeled UF and weighing factors table. There is no AQoS module proposed as in Fig. 17 in literature to the best of our knowledge.

If the AQoS descriptor of a video sequence is required to be prepared using the AQoS module, the video sequence is first categorized into a class using the ML and SFL classification blocks of the framework. Then, the weighing factors table is used to determine the weights associated with its class, and these weights are assigned to the developed UF in the UF generator block. Simultaneously, the video sequence is encoded to produce sub-streams with different scalability parameters. After encoding, the bit stream extractor tool of JSVM [JSVM 9.13.1 Software] is used to get necessary details for the adaptation parameter values of scalable sub-streams. The quality parameter values of these sub-streams are measured with VQM [Wolf et. al., 2002]. In order to measure VQM grades of the sub-streams that have different spatial resolutions and frame rates, the original-uncompressed versions of the video sequences were spatially and/or temporally down-sampled. The UF including the assigned weights, the scalability/adaptation parameter values of the sub-streams, and the VQM grades of the quality parameters of the sub-streams are input to the AQoS generator to produce the AQoS descriptor. The UCD module supplies the bandwidth of the network and the width 
and height of the user display constraints in this work. After the ADTE determines the most appropriate adaptation parameter values, they are passed to the AE to generate the adapted scalable bit stream, as decided.

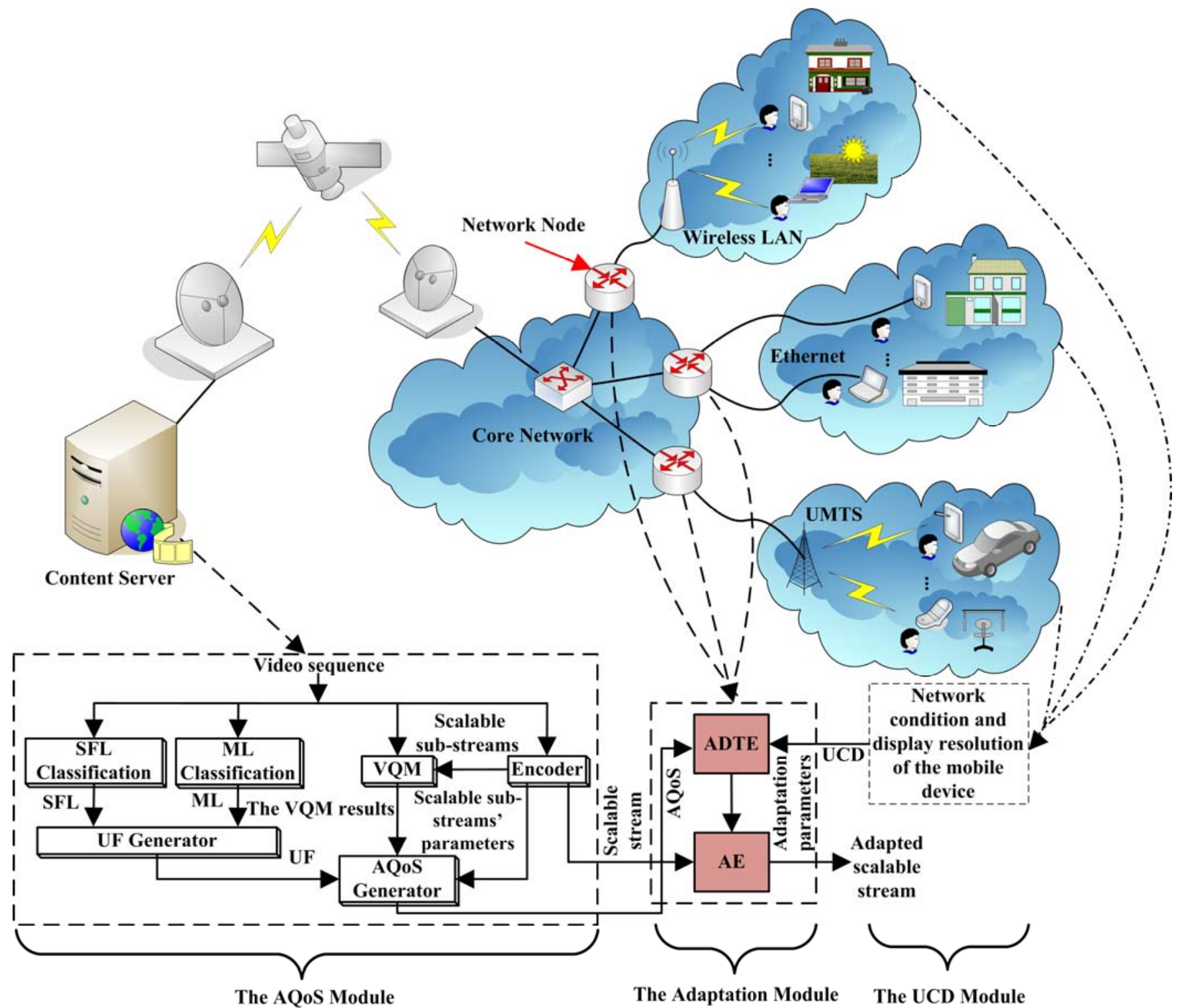

Fig. 17. The architecture for the proposed adaptation decision taking framework

\section{ADAPTATION DECISION TAKING PROCESS AND RESULTS TO EVALUATE THE PERFORMANCE OF THE}

PROPOSED FRAMEWORK

The ADTE shown in Fig. 17 determines the most appropriate adaptation parameters by solving the optimization problem given below:

Maximize: $\left\{U F=W_{F} F+W_{S} S+W_{Q} Q\right\}=>$ Optimization Constraint 
Subject to: $\{$ bit rate $\leq$ target bit rate; width $\leq$ display width; height $\leq$ display height $\}=>$ Limitation Constraint

where the optimization constraint represents maximization of the UF (see (4)), bit rate is the bit rate of the supported video streams, target bit rate is the network bandwidth, width and height are the horizontal and vertical dimensions of the supported sub-scalable video stream resolutions. Display width and height are the horizontal and vertical dimensions of the terminal display resolution that will be used to consume the adapted bit stream, respectively.

Fig. 18 illustrates the flowchart of the decision process. In order to assess the performance of the proposed adaptation decision taking framework, a set of experiments was carried out. The test sequences used in the experiments were encoded with three spatial (i.e., QCIF, CIF, and SD), four temporal (i.e., 3.75, 7.5, 15, and $30 \mathrm{fps}$ ), and two Medium Grain Scalability (MGS) [Thang et. al., 2004] layers added to every spatial enhancement layer. JSVM 9.13.1 [JSVM 9.13.1] was used as the reference software for the encoding process. A set of test sequences, namely: News, Big Ships, Coastguard, Football, Silent, Bus, Tempete, and Tennis, was used to evaluate the performance of the proposed adaptation decision taking concept. These sequences contain the frames of the associated entire test sequences ranging from $0-150,70-220,100-250,0-150,0-150,15-165,0-150$, and $0-150$, respectively. The classes of the test sequences are determined by the assistance of the proposed classification algorithms as $1,2,3,4,5,6,7$, and 8 , respectively. The adopted UCD for the experiments consists of the following limitation constraints: the network bandwidths $=\{200,500,700$, and 1000$\}$ kbps selected for mobile devices, display width $=704$ pixels, and display height $=576$ pixels. It has to be noted that before any of the constraints restrict the adaptation decisions, the scalable sub-stream parameters described in the AQoS are identified as the candidate adaptation decision results. During the adaptation decision taking process, first of all, the limitation constraints (i.e., the network bandwidth and display resolution) were considered. Accordingly, the sub-streams that have higher bit rates than the target bit rate were eliminated from the candidate adaptation decisions. Then, the sub-streams that have higher spatial resolutions than the display resolution were removed from the candidates. Following, the optimization constraint was applied to the remaining candidate adaptation decisions. The candidate adaptation decisions that yield the maximum UF output were designated as the optimal adaptation decisions. 


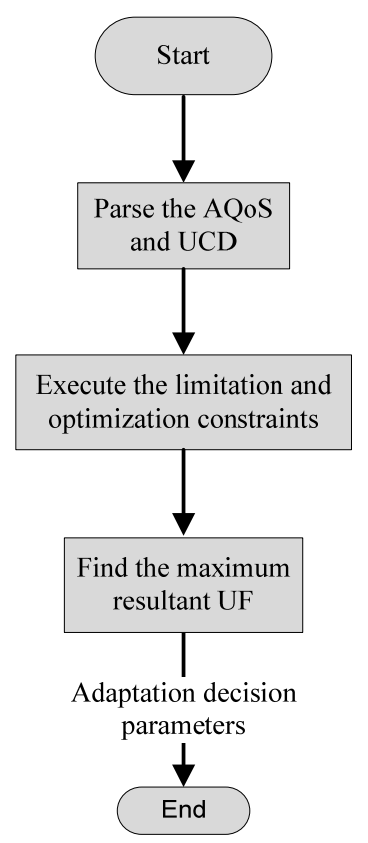

Fig. 18. The flowchart of the adaptation decision taking strategy

Table VII presents the experimental results of the proposed ADTE. The resultant bit rates, spatial resolutions, frame rates, and VQM grades after taking the adaptation decisions are shown in this table. In order to compare the performance of the proposed adaptation decision taking results, the adaptation decision taking algorithms of [Mukherjee et. al., 2005] and [Prangl et. al., 2007] were also implemented as the references for the benchmarking. The test sequences were subjected to the same encoding specifications for the reference techniques. Moreover, the same sequences were also used for fair comparison purposes. The reason behind using the approach discussed in [Mukherjee et. al., 2005] as a reference technique is that it relies on a basic adaptation decision taking method. A simple UF, which only considers bit rate of video contents and is irrespective of video content characteristics, is utilized in [Mukherjee et. al., 2005]. The reason behind using the method discussed in [Prangl et. al., 2007] is that the adaptation decision taking algorithm in this reference work also relies on a designed UF that considers frame rate, spatial resolution, and QP of a scalable video sequence. The importance of the spatial resolution, frame rate, and QP on the total utility value is calculated using weights. The weights of the UF for each user are determined using a recommendation system to maintain feedback from the user in [Prangl et. al., 2007]. The following parameters: width $_{\text {original }}=704$, height ${ }_{\text {original }}=576$, framerate $_{\text {original }}=30 \mathrm{fps}, \mathrm{QP}_{\min }=30$, and $\mathrm{QP}_{\max }=35$ were defined to apply the reference UF. Moreover, in order to fairly compare the reference and the proposed adaptation decision taking techniques, the reference method is implemented using the same test sequences to determine the UF weights for each of the classes. The determined weights of the 
reference work are illustrated in Table VIII.

It should be noted that the characteristics of video contents are not considered in adaptation decision taking operations of the reference methods, which are believed to affect the decisions taken as demonstrated in the proposed study. Table VII also illustrates the results of the reference experiments. The adaptation decision taking results obtained using the proposed UF, the reference study in [Mukherjee et. al., 2005] and [Prangl et. al., 2007] are illustrated with p.1, r.1, and r.2, respectively, in the table. Using the adaptation decision taking results of the proposed and reference techniques, adaptation experiments were performed, and subjective tests were conducted to evaluate the adapted sequences with human observers. The DSIS method [ITU-Recommendation BT.500-11, 2002] was employed for the subjective tests. 18 viewers participated in the experiments. After the outliers were detected, using the outlier screening method in [ITU-Recommendation BT.500-10, 2000], and removed, 16 observers were used to calculate the MOS results in the experiments. The MOSs of the subjective tests are shown on the right-hand side of Table VII together with $95 \%$ confidence intervals.

As can be observed from the results, when the network bandwidth is $200 \mathrm{kbps}$, the adaptation parameter values determined by the proposed ADTE for the News sequence are specified as bit rate: $179 \mathrm{kbps}$, spatial resolution: CIF, frame rate: 7.5 fps, SNR: 1, and VQM grade: 4.21. A MOS of 4.12 is achieved using the proposed adaptation decision taking technique. However, the adaptation parameters values determined by the reference techniques are: bit rate: $199 \mathrm{kbps}$, spatial resolution: CIF, frame rate: $3.75 \mathrm{fps}$, and SNR: 2. A MOS of 3.93 is attained by utilizing the reference techniques in adaptation decision taking process. Even though the bit rate of the News sequence adapted using the adaptation parameter values determined by the proposed ADTE is lower than that by the reference techniques, the MOS score (i.e., 4.12) achieved by the proposed ADTE is higher than that by the reference ADTEs (i.e., 3.93). The reason is that the video quality perception saturates at lower frame rates due to high frame quality, so it is enhanced with higher frame rate.

As can also be seen from the results, when the network bandwidth is $500 \mathrm{kbps}$, a bit rate, spatial resolution, frame rate, SNR, and VQM grade of $499 \mathrm{kbps}, \mathrm{SD}, 7.5 \mathrm{kbps}, 0,4.11$, respectively, can be achieved by utilizing the proposed method and reference study in [Mukherjee et. al., 2005] in adaptation decision taking process. A MOS of 4.25 is attained using both techniques. Nevertheless, a bit rate, spatial resolution, frame rate, and SNR of $430 \mathrm{kbps}, \mathrm{SD}$, $3.75 \mathrm{kbps}, 1$, respectively, can be achieved using the reference study in [Prangl et. al., 2007] in adaptation decision 
taking process, which results in a MOS value of 4.19.

It has to be noted that, the VQM results of the sequences are only presented for the proposed technique in Table VII since VQM is only used in the proposed technique to compute quality parameters of the sequences.

Similar results can be observed from all of the remaining MOS results throughout the table. Thus, it can be concluded that the proposed technique either outperforms the reference techniques or present similar results for the adapted sequences. Even though the proposed technique sometimes presents similar results to the reference techniques, the outperforming results emphasize the efficiency of the proposed technique.

In order to present the performance of the proposed technique, the adaptation results are also evaluated using SSIM as illustrated in Table VII. Using these SSIM results, the readers who are familiar with objective quality evaluation techniques can get clearer idea about the performance of the results. It should be noted that subjective quality evaluation technique presents results

In order to adapt a new video sequence, the information related to this sequence is offline prepared by the AQoS descriptor, which is in the AQoS module of Fig. 17. Using this offline information, the proposed framework becomes quite time efficient and quick to adapt the video sequence.

It is tested that classifying video sequences more than into eight classes does not provide different adaptation decision taking results than having eight classes. Thus, it can be envisaged that the ML and SFL classification algorithms are sufficient enough to efficiently decide on the best spatial resolution, frame rate, and quality parameters for adapting video streams. 
TABLE VII

COMPARISON OF THE PROPOSED ADAPTATION DECISION TAKING RESULTS WITH THE REFERENCE TECHNIQUES FOR DIFFERENT VIDEO SEQUENCES

\begin{tabular}{|c|c|c|c|c|c|c|c|c|c|c|c|c|c|c|c|c|c|c|c|c|}
\hline \multirow{2}{*}{ 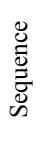 } & \multirow{2}{*}{ 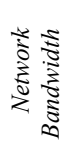 } & \multicolumn{3}{|c|}{ Bit Rate (kbps) } & \multicolumn{3}{|c|}{ Spatial Resolution } & \multicolumn{3}{|c|}{ Frame Rate (fps) } & $V Q M$ & \multicolumn{3}{|c|}{$S N R$} & \multicolumn{3}{|c|}{ MOS-Confidence Interval } & \multicolumn{3}{|c|}{ SSIM } \\
\hline & & p.1 & r. 1 & $\mathrm{r} .2$ & p.1 & r. 1 & r.2 & p.1 & r.1 & $\mathrm{r} .2$ & p.1 & p. 1 & r. 1 & r. 2 & p.1 & r.1 & r. 2 & p.1 & r. 1 & $\mathrm{r} .2$ \\
\hline \multirow{4}{*}{$\sum_{0}^{\infty}$} & 200 & 179 & 199 & 199 & $\mathrm{CIF}$ & $\mathrm{CIF}$ & $\mathrm{CIF}$ & 7.5 & 3.75 & 3.75 & 4.21 & 1 & 2 & 2 & $4.12 \pm 0.31$ & $3.93 \pm 0.42$ & $3.93 \pm 0.42$ & 0.955 & 0.964 & 0.964 \\
\hline & 500 & 499 & 499 & 430 & SD & SD & SD & 7.5 & 7.5 & 3.75 & 4.11 & 0 & 0 & 1 & $4.25 \pm 0.35$ & $4.25 \pm 0.35$ & $4.19 \pm 0.29$ & 0.946 & 0.946 & 0.945 \\
\hline & 700 & 551 & 632 & 621 & SD & SD & SD & 7.5 & 15 & 3.75 & 4.28 & 1 & 0 & 2 & $4.44 \pm 0.31$ & $4.31 \pm 0.29$ & $4.38 \pm 0.38$ & 0.967 & 0.944 & 0.965 \\
\hline & 1000 & 932 & 932 & 932 & SD & SD & SD & 7.5 & 7.5 & 7.5 & 4.57 & 2 & 2 & 2 & $4.56 \pm 0.24$ & $4.56 \pm 0.24$ & $4.56 \pm 0.24$ & 0.969 & 0.969 & 0.969 \\
\hline \multirow{4}{*}{ 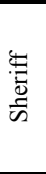 } & 200 & 192 & 192 & 148 & QCIF & QCIF & QCIF & 7.5 & 7.5 & 3.75 & 4.49 & 1 & 1 & 2 & $3.56 \pm 0.36$ & $3.56 \pm 0.37$ & $3.37 \pm 0.31$ & 0.896 & 0.896 & 0.920 \\
\hline & 500 & 467 & 498 & 425 & CIF & QCIF & CIF & 7.5 & 15 & 3.75 & 4.53 & 1 & 2 & 2 & $3.87 \pm 0.30$ & $3.50 \pm 0.31$ & $3.43 \pm 0.42$ & 0.887 & 0.914 & 0.926 \\
\hline & 700 & 584 & 699 & 584 & CIF & QCIF & $\mathrm{CIF}$ & 7.5 & 30 & 7.5 & 4.90 & 2 & 1 & 2 & $3.93 \pm 0.42$ & $3.75 \pm 0.33$ & $3.93 \pm 0.42$ & 0.910 & 0.891 & 0.910 \\
\hline & 1000 & 965 & 998 & 998 & SD & SD & SD & 7.5 & 3.75 & 3.75 & 4.82 & 1 & 2 & 2 & $4.12 \pm 0.42$ & $4.06 \pm 0.34$ & $4.06 \pm 0.34$ & 0.937 & 0.951 & 0.951 \\
\hline \multirow{4}{*}{ 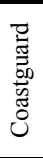 } & 200 & 158 & 158 & 133 & QCIF & QCIF & QCIF & 15 & 15 & 30 & 3.96 & 2 & 2 & 1 & $2.94 \pm 0.33$ & $2.94 \pm 0.33$ & $2.87 \pm 0.27$ & 0.942 & 0.942 & 0.919 \\
\hline & 500 & 452 & 492 & 492 & CIF & CIF & CIF & 7.5 & 15 & 15 & 3.18 & 0 & 1 & 1 & $3.12 \pm 0.30$ & $3.06 \pm 0.35$ & $3.06 \pm 0.35$ & 0.884 & 0.911 & 0.911 \\
\hline & 700 & 575 & 642 & 575 & CIF & CIF & CIF & 30 & 7.5 & 30 & 3.16 & 0 & 1 & 0 & $3.31 \pm 0.29$ & $3.19 \pm 0.27$ & $3.31 \pm 0.29$ & 0.876 & 0.915 & 0.876 \\
\hline & 1000 & 911 & 999 & 911 & CIF & SD & CIF & 30 & 3.75 & 30 & 3.64 & 1 & 0 & 1 & $3.50 \pm 0.25$ & $3.06 \pm 0.22$ & $3.50 \pm 0.25$ & 0.908 & 0.890 & 0.908 \\
\hline \multirow{4}{*}{$\begin{array}{l}\overline{\bar{\tau}} \\
\overline{0} \\
0 \\
0\end{array}$} & 200 & 191 & 191 & 191 & QCIF & QCIF & QCIF & 15 & 15 & 15 & 3.53 & 0 & 0 & 0 & $2.81 \pm 0.27$ & $2.81 \pm 0.27$ & $2.81 \pm 0.27$ & 0.883 & 0.883 & 0.883 \\
\hline & 500 & 404 & 497 & 404 & QCIF & CIF & QCIF & 30 & 3.75 & 30 & 3.89 & 1 & 0 & 1 & $3.31 \pm 0.29$ & $2.69 \pm 0.34$ & $3.31 \pm 0.29$ & 0.914 & 0.861 & 0.914 \\
\hline & 700 & 513 & 623 & 404 & QCIF & CIF & QCIF & 30 & 7.5 & 30 & 3.95 & 2 & 0 & 1 & $3.37 \pm 0.27$ & $3.06 \pm 0.22$ & $3.31 \pm 0.29$ & 0.932 & 0.859 & 0.914 \\
\hline & 1000 & 908 & 943 & 908 & CIF & CIF & $\mathrm{CIF}$ & 15 & 7.5 & 15 & 3.49 & 0 & 2 & 0 & $3.62 \pm 0.35$ & $3.43 \pm 0.40$ & $3.62 \pm 0.35$ & 0.856 & 0.940 & 0.856 \\
\hline \multirow{4}{*}{$\underset{\overrightarrow{0}}{\overrightarrow{0}}$} & 200 & 189 & 189 & 189 & $\mathrm{CIF}$ & CIF & CIF & 7.5 & 7.5 & 7.5 & 3.57 & 0 & 0 & 0 & $3.56 \pm 0.31$ & $3.56 \pm 0.31$ & $3.56 \pm 0.31$ & 0.928 & 0.928 & 0.928 \\
\hline & 500 & 430 & 431 & 430 & CIF & CIF & CIF & 7.5 & 30 & 7.5 & 3.93 & 2 & 1 & 2 & $3.87 \pm 0.43$ & $3.48 \pm 0.47$ & $3.87 \pm 0.43$ & 0.948 & 0.940 & 0.948 \\
\hline & 700 & 648 & 648 & 648 & SD & SD & SD & 7.5 & 7.5 & 7.5 & 3.98 & 1 & 1 & 1 & $3.91 \pm 0.38$ & $3.91 \pm 0.38$ & $3.91 \pm 0.38$ & 0.924 & 0.924 & 0.924 \\
\hline & 1000 & 806 & 972 & 972 & SD & SD & SD & 15 & 7.5 & 7.5 & 3.88 & 1 & 2 & 2 & $4.12 \pm 0.39$ & $4.06 \pm 0.44$ & $4.06 \pm 0.44$ & 0.923 & 0.947 & 0.947 \\
\hline \multirow{4}{*}{$\begin{array}{l}\stackrel{2}{\pi} \\
\text { की } \\
.00\end{array}$} & 200 & 154 & 162 & 145 & CIF & CIF & CIF & 15 & 30 & 7.5 & 3.17 & 0 & 0 & 0 & $3.56 \pm 0.41$ & $3.50 \pm 0.44$ & $3.43 \pm 0.36$ & 0.840 & 0.836 & 0.844 \\
\hline & 500 & 383 & 396 & 335 & $\mathrm{CIF}$ & CIF & $\mathrm{CIF}$ & 15 & 30 & 3.75 & 4.35 & 2 & 2 & 2 & $3.75 \pm 0.32$ & $3.62 \pm 0.39$ & $3.56 \pm 0.40$ & 0.893 & 0.889 & 0.914 \\
\hline & 700 & 687 & 687 & 622 & SD & SD & SD & 15 & 15 & 7.5 & 4.12 & 1 & 1 & 1 & $3.93 \pm 0.45$ & $3.93 \pm 0.45$ & $3.81 \pm 0.33$ & 0.933 & 0.933 & 0.934 \\
\hline & 1000 & 929 & 992 & 913 & SD & SD & SD & 7.5 & 15 & 3.75 & 4.78 & 2 & 2 & 2 & $4.37 \pm 0.42$ & $4.25 \pm 0.31$ & $4.12 \pm 0.27$ & 0.947 & 0.945 & 0.941 \\
\hline \multirow{4}{*}{ 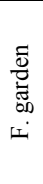 } & 200 & 152 & 191 & 152 & QCIF & QCIF & QCIF & 30 & 15 & 30 & 4.25 & 1 & 2 & 1 & $2.81 \pm 0.37$ & $2.68 \pm 0.38$ & $2.81 \pm 0.37$ & 0.907 & 0.930 & 0.907 \\
\hline & 500 & 220 & 454 & 152 & QCIF & CIF & QCIF & 30 & 3.75 & 30 & 4.56 & 2 & 0 & 1 & $3.18 \pm 0.30$ & $3.00 \pm 0.43$ & $2.81 \pm 0.37$ & 0.928 & 0.942 & 0.907 \\
\hline & 700 & 640 & 677 & 640 & $\mathrm{CIF}$ & CIF & CIF & 15 & 7.5 & 15 & 3.67 & 0 & 1 & 0 & $3.56 \pm 0.40$ & $3.31 \pm 0.34$ & $3.56 \pm 0.40$ & 0.868 & 0.903 & 0.868 \\
\hline & 1000 & 963 & 963 & 963 & CIF & CIF & CIF & 30 & 30 & 30 & 4.24 & 1 & 1 & 1 & $3.93 \pm 0.33$ & $3.93 \pm 0.33$ & $3.93 \pm 0.33$ & 0.896 & 0.896 & 0.896 \\
\hline \multirow{4}{*}{$\stackrel{n}{\Xi}$} & 200 & 192 & 192 & 148 & QCIF & QCIF & QCIF & 30 & 30 & 30 & 4.10 & 2 & 2 & 1 & $3.25 \pm 0.38$ & $3.25 \pm 0.38$ & $3.06 \pm 0.34$ & 0.920 & 0.920 & 0.902 \\
\hline & 500 & 415 & 494 & 415 & CIF & $\mathrm{CIF}$ & $\mathrm{CIF}$ & 15 & 3.75 & 15 & 3.64 & 0 & 1 & 0 & $3.37 \pm 0.47$ & $2.75 \pm 0.38$ & $3.37 \pm 0.47$ & 0.844 & 0.890 & 0.844 \\
\hline & 700 & 507 & 638 & 507 & CIF & $\mathrm{CIF}$ & CIF & 30 & 7.5 & 30 & 3.70 & 0 & 2 & 0 & $3.68 \pm 0.44$ & $3.50 \pm 0.25$ & $3.68 \pm 0.44$ & 0.842 & 0.922 & 0.842 \\
\hline & 1000 & 968 & 968 & 777 & CIF & CIF & CIF & 30 & 30 & 30 & 4.24 & 2 & 2 & 1 & $4.06 \pm 0.54$ & $4.06 \pm 0.54$ & $3.81 \pm 0.35$ & 0.922 & 0.922 & 0.896 \\
\hline
\end{tabular}

TABLE VIII

THE WEIGHTS OF THE REFERENCE ADAPTATION DECISION TAKING TECHNIQUE DISCUSSED IN [PRANGL ET. AL., 2007]

\begin{tabular}{|c|c|c|c|}
\hline \multirow{2}{*}{ Class } & \multicolumn{3}{|c|}{ UF Weights } \\
\cline { 2 - 4 } & $W_{F}$ & $W_{S}$ & $W_{O}$ \\
\hline 1 & 0.00 & 0.60 & 0.40 \\
\hline 2 & 0.20 & 0.40 & 0.40 \\
\hline 3 & 0.60 & 0.20 & 0.20 \\
\hline 4 & 0.80 & 0.10 & 0.10 \\
\hline 5 & 0.00 & 0.70 & 0.30 \\
\hline 6 & 0.10 & 0.50 & 0.40 \\
\hline 7 & 0.60 & 0.20 & 0.20 \\
\hline 8 & 0.80 & 0.10 & 0.10 \\
\hline
\end{tabular}




\section{CONCLUSIONS AND FUTURE WORK}

In this paper, an advanced video adaptation decision taking technique relying on a utility-based approach has been proposed to select the most adequate frame rate, spatial resolution, and quality parameters to enable seamless video access for mobile devices. In order to assist this technique, a UF model that considers the correlations of frame rate, spatial resolution, and quality parameters has also been proposed to decide on the best set of scalability parameters. A video adaptation framework has been developed to perform the proposed video adaptation decision taking technique and adaptation operations. The MPEG-21 supported tools (i.e., AQoS and UCD) have been used to assist the adaptation decision taking process of the framework, so as to better align the operation of the proposed architecture to the international standards. Motion and structural feature level driven classification methods have been developed to categorize the video contents into distinctive types. Using video contents belonging to different categories, subjective experiments have been carried out. A table of weighing factors has been designed based on the knowledge gained through the subjective tests. Accordingly, the weights of the UF have been determined depending on the video content type. It is envisaged that the work presented in this paper will lead to wide-spread seamless and adaptive video access for mobile devices, which in turn will enable improved video perception of users.

\section{REFERENCES}

Akyol E., Tekalp A. M., and Civanlar M. R., 2007, "Content-aware Scalability-type Selection for Rate Adaptation of Scalable Video,” EURASIP Journ. on Applied Signal Proc. 2007, Article ID 10236.

Bocheck P., Nakajima Y., and Chang S.-F., 1999, "Real-time Estimation of Subjective Utility Functions for MPEG-4 Video Objects," Proc. of IEEE Packet Video Workshop, New York, USA, Apr.

Bouguet J.Y., 1999 "Pyramidal Implementation of the Lucas Kanade Feature Tracker Description of the Algorithm," OpenCV Documentation, Intel Corporation, Microprocessor Research Labs.

Canny J.F., "A Computational Approach to Edge Detection," IEEE Trans. Pat. Analysis and Machine Intelligence," no. 8, pp. 679-698, 1986.

Carvalho, P., 2004, "Multimedia Content Adaptation for Universal Access," M.Sc. Thesis, Faculdade de Engenharia da Universidade do Porto, Portugal.

Chang S. F., 2002, “Optimal Video Adaptation and Skimming Using a Utility-Based Framework," Tyrrhenian Int. Workshop on Digital Communications, Capri Island, Italy, Sep. 
Chang S. F., and Vetro A., 2005, "Video Adaptation: Concepts, Technologies and Open Issues," Proc. IEEE Proc. Special Issue on Advances in Video Coding and Delivery, vol. 93, no. 1, pp. 148-158, Jan.

Devore J. L., 1995 “Probability and Statistics for Engineering and the Sciences”, $4^{\text {th }}$ Edition, pp. 474-585, Duxbury.

Douglas A., 1971, "Examples Concerning Efficient Strategies for Gaussian Elimination,” vol. 8, pp. 3-4, Springer, Sep.

Eberhard M., Celetto L., Timmerer C., Quacchio E., and Hellwagner H., 2007, "Performance Analysis of Scalable Video Adaptation: Generic versus Specific Approach," IEEE Trans. on CSVT, vol. 17, no. 9, pp. 50-53.

Fleet D.J., and Wiess Y., 2006, "Optical flow estimation in paragios," Handbook of Math. Models in Comput. Vision, Springer, pp. 239.

Grigorescu C., Perkov N., and Westenberg M. A., 2004, "Contour and Boundary Detection Improved by Surround Suppression of Texture Edges,” Journal of Image and Vision Computing, vol. 22, pp. 583-679, Aug.

Hands D. S., 2004, “A Basic Multimedia Quality Model,” IEEE Trans. Multimedia, vol. 6, no. 6, Dec.

Hsiao M. H., Chen Y. W., Chen H. T., Chou K. H., and Lee S. Y., 2007, "Content-Aware Video Adaptation under Low-Bitrate Constraint," EURASIP Journal on Advances in Signal Processing, vol. 2007, Article ID 17179, May.

Information Technology- Multimedia Frameworks-Part 7: Digital Item Adaptation," ISOIIEC Standard ISO-IEC 21000-7:2007, Dec. 2007.

JSVM 9.13.1 Software, downloaded from CVS Server, garcon.ient.rwth-aachen.de/cvs/jv

Lucas B. D. and Kanade T., 1981, "An Iterative Image Registration Technique with an Application to Stereo Vision,” Int. Joint Conf. Artificial Intell. pp. 674-679.

Mangasarian O.L. and Wild E.W., 2004, "Feature Selection in K-median Clustering," Workshop on Clustering High Dimensional Data and its Applications, pp. 23-28, La Buena Vista, FL.

Methodology for the Subjective Assessment of the Quality of Television Pictures, ITU-Recommendation BT.500-11, 2002. [Online]. Available: http://www.itu.org

Methodology for the Subjective Assessment of the Quality of Television Pictures, ITU-Recommendation BT.500-10, 2000, [Online]. Available: http://www.itu.org

Mukherjee D., Delfosse E., Kim J-G., and Wang Y., "Optimal Adaptation Decision Taking for Terminal and Network Quality of Service,”IEEE Trans. on Multimedia, vol. 7, no. 7, pp. 454-462, Jun. 2005.

Onur O. D. and Alatan A. A., 2007, "Video Adaptation Based on Content Characteristics and Hardware Capabilities," Second Int. Workshop on Semantic Media Adaptation and Personalization, pp. 15-20, Uxbridge, UK, 17-18 Dec. 
Onur O. D. and Alatan A. A., 2005, "Video Adaptation for Transmission Channels by Utility Modeling," IEEE Int. Conf. on Multimedia and Expo, Amsterdam, The Netherlands, 6 Jul.

Papari G., Campisi P., Petkov N., and Neri A., 2006, “A Multiscale Approach to Contour Detection by Texture Suppression," SPIE Image Proc.: Alg. and Syst., San Jose, CA, USA, Jan.

Prangl M., Szkaliczki T., Hellwagner H., 2007, “A Framework for Utility-Based Multimedia Adaptation,” IEEE Trans. on Circuits and Systems for Video Technology, Vol. 17, No. 6, Jun.

Pinson M.H. and Wolf S., 2004, “A New Standardized Method for Objectively Measuring Video Quality,” IEEE Transactions on Broadcasting, vol. 50, no.3, pp. 312-322, Sep.

Sofokleous A. A. and Angelides M. C., 2008, "DCAF: An MPEG-21 Dynamic Content Adaptation Framework," Springer Multimedia Tools Applications, vol. 40, no. 2, pp. 151-182, Mar.

Shechtman E., Caspi Y., Irani M., 2005, "Space-time super-resolution,” IEEE Trans. on Pattern Analysis and Machine Intelligence, no. 27, vol. 4, pp. 531-545.

Thang, C., Kim, J.-G., Kang, J.W., and Yoo, J.-J., 2009, "SVC Adaptation: Standard Tools and Supporting Methods,” Elsevier Signal Processing: Image Communication, vol. 24, pp. 214-228.

Tomasi C. and Kanade T., "Detection and Tracking of Point Features," Carnegie Mellon University Technical Report CMU-CS-91-132, April 1991.

Vetro A., "MPEG-21 Digital Item Adaptation: Enabling Universal Multimedia Access," IEEE Transactions on Multimedia, vol. 11, issue 1, pp. 84-87, Mar. 2004.

Yamazaki T., "Subjective Video Quality Assessment for Adaptive Quality of Service Control," IEEE Int. Conf. Multimedia and Expo, Tokyo, Japan, Aug. 2001.

Wang Z., Bovik A., Sheikh H., and Simoncelli E., 2004, "Image Quality Assessment: from Error Visibility to Structural Similarity," IEEE Trans. Image Proc., pp. 600-612.

Wang Y., Kim J.-G., Chang S.-F., and Kim H.-M., 2007, “Utility-Based Video Adaptation for Universal Multimedia Access (UMA) and Content-Based Utility Function Prediction for Real-Time Video Transcoding, IEEE Transactions on Multimedia, vol. 9, pp. 213-220, Feb.

Wolf S. and Pinson M., 2002,"VQM Software and Measurement Techniques," National Telecommunications and Information Administration (NTIA'02) Report 02-392, Jun. 